\title{
Abschliessende Gedanken
}

Meine Analyse, welche Aspekte bewussten Erlebens unter Berücksichtigung des Desiderats phänomenaler Adäquatheit unbedingt noch in die philosophische Debatte einfliessen müssten, soll zu einer Neueinschätzung des hard problem beitragen, die dessen Explanandum hinreichend berücksichtigt und danach fragt, welche Phänomene eigentlich zur Debatte stehen. ${ }^{1}$ Aufgrund der hier aufgezeigten Ubiquität von Qualia bzw. phänomenalem Erleben, lässt sich konstatieren, dass das Explanandum des hard problem nicht nur in marginalen, eigenartigen Formen des Erlebens besteht, sondern in den Phänomenen des >Bewusstseins< bzw. des >Erlebens< überhaupt. Infolgedessen müssen diese, für eine Theorie des Geistes zentralen Phänomene, auch in einer Weise, die phänomenal adäquat ist, berücksichtigt werden. Eine Analyse des hard problem, die diesem Desiderat gerecht wird, besteht deshalb nicht in erster Linie in der Beantwortung von Fragen aus nicht-phänomenalen Problemoptiken, wie z.B., ob bewusstes Erleben kausal relevant ist, mit welchen Phänomenen es korreliert, ob es mit seinen Korrelaten irgendwie sidentisch< sein könnte oder ob Phänomenales nur >scheinbar ${ }^{2}$ existiert oder nicht etc. ${ }^{3}$ Denn mit welchen Phänomenen Erleben korreliert oder ob die erlebten Gehalte veridisch oder nur sscheinbar sind, stellen gänzlich andere Fragen dar, als die, warum es irgendwie ist, zu erleben; und gerade darin, dass einem Subjekt etwas >erscheint<, dass es für einen mit Bewusstsein ausgestatteten Organismus irgendwie ist, wenn für ihn etwas >scheinbar< ist, besteht das Phänomenale bzw. das im hard problem zur Debatte stehende Explanandum.

\subsection{Funktionen verengter und transformierter Begriffe des Phänomenalen}

Die in den Kapiteln 8 und 9 ausgewiesene Notwendigkeit eines erweiterten Qualia-Begriffs führt deshalb zu einer Neueinschätzung des hard problem,

1 Vgl. dazu auch Chalmers, David J.: The conscious mind. In search of a fundamental theory, New York: Oxford University Press 1996, S. xi-xiv.

2 Zu dieser Behauptung vgl. Dennett, Daniel C.: »Lovely and Suspect Qualities«, 1991, Online: http://cogprints.org/263/1/lovely\%26s.htm [zuletzt geprüft am 24.09.2018].

3 Vgl. dazu auch Abschnitt 5 mit der Beschreibung von sechs Problemoptiken 
weil klar wird, dass letzteres nicht mit phänomenal inadäquaten QualiaKonzeptionen entschärft werden kann. Wenn also beispielsweise Dennett in seinem Aufsatz »Quining Qualia « ${ }^{4}$ versucht, Qualia zu »eliminieren «, genauer aber nur seinen eigenen, engen Qualia-Begriff widerlegt, ${ }^{6}$ so ist damit das hard problem nicht einmal im Ansatz weniger hart geworden. Dennett gibt sogar zu, dass sein Versuch, Qualia zu >eliminieren<, nicht so sehr eine Doktrin, sondern eine Taktik (»tactical issue «) $)^{7}$ sei, um der Verwirrung im Zusammenhang mit Qualia zu begegnen. Er begegnet aber dieser Verwirrung, mit einer weiteren Verwirrung, indem er auf einen phänomenal inadäquaten QualiaBegriff rekurriert, um dann Qualia in toto eliminieren zu können. ${ }^{8}$ Diese Taktik kann (und will) natürlich nicht dem Desiderat phänomenal Adäquatheit gerecht werden, sondern dient wohl eher dem Ziel, zu >zeigen<, dass es keine Qualia >gibt<.

Daher drängt sich die Vermutung auf, dass ein Qualia-Begriff, der vor allem auf umstrittenen, marginalen, seltsamen Eigenschaften und zudem auf einer verengten Auswahl von Erlebnismöglichkeiten beruht, die Funktion haben könnte, sich dem Explanandum nicht in seiner vollständigen Problemdimension, sondern nur in zweifelhaften Aspekten davon, stellen zu müssen. Deshalb könnte die Funktion des Qualia-Begriffs im Diskurs um das hard problem nicht nur, wie Crane feststellt, darin liegen, dass Reduktionsskeptiker/innen mit ihm aufzuzeigen versuchen, dass mit reduktionistisch-physikalistischen Ansätzen, auch wenn sie sich für das easy problem ${ }^{9}$ als brauchbar erweisen, Qualia nicht hinreichend zu beschreiben bzw. zu erklären sind. ${ }^{10}$ Die in der Literatur vorgefundenen, nicht selten ziemlich verengten und phänomenal inadäquaten Qualia-Begriffe weisen vielmehr darauf hin, dass bestimmte Vorentscheidungen zum Qualia-Begriff, die mit einer Auswahl von angeblichen und auch besonders seltsamen 〉Qualia-Eigenschaften< einhergehen, auch die Funktion einer leicht zu treffenden Zielscheibe von Qualia-Skeptikern/innen erfüllen könnte, um darzulegen, dass es phänomenales Erleben, so konzipiert, nicht >gibt<. Mit einem solchen Qualia-Begriff ist es dann ein Leichtes, die

4 Dennett, Daniel C.: »Quining Qualia«, in: Philosophy of mind. Classical and contemporary readings, Chalmers, David J. [Hrsg.], New York: Oxford University Press [zuerst 1988]/2002.

5 A.a.O., S. 244, [Fussnote Nr. 2].

6 Vgl. dazu Abschnitt 9.1

7 Ebd.

8 Vgl. a.a.O., S. 229 .

9 Vgl. Chalmers, David J.: The conscious mind. In search of a fundamental theory, New York: Oxford University Press 1996, S. 25 sowie 106-107.

10 Vgl. dazu auch Crane, Tim: »The origins of qualia«, 2000, Online: http://sas-space.sas. ac.uk/220/1/The\%2oorigins\%20of\%2oqualia.pdf [zuletzt geprüft am 24.09.2018], S. 22. 
>Elimination< von Qualia zu feiern: »[...] contrary to what seems obvious at first blush there simply are no qualia at all «.11

Zum anderen könnte die Funktion von transformierten bzw. phänomenal inadäquaten Qualia-Begriffen darin liegen, dass sich reduktionsoptimistische Positionen nur noch um das easy problem kümmern müssen, weil sie auf dieser Grundlage behaupten können, dass phänomenales Erleben, so konzipiert, >identisch< mit physikalischen Eigenschaften ist. ${ }^{12}$ Insbesondere vor dem Hintergrund einer unauflösbar scheinenden Patt-Situation ${ }^{13}$ und der gegenseitigen Beweislastzuschiebung zwischen reduktionsoptimistischen und reduktionsskeptischen Positionen kann demnach ein phänomenal inadäquater Qualia-Begriff die Funktion einer der Explizierbarkeit dienlichen Transformation des Phänomenalen erfüllen (vgl. Abschnitt 7.2) oder, wie in den Abschnitten 7.1, 8.1 und 9.2 dargestellt, als vermeintliche Grundlage für eine $>$ Elimination $<$ des Explanandums dienen.

Mit anderen Worten: die Funktion von phänomenal inadäquaten und verengten Qualia-Begriffen könnte sogar darin bestehen, einer Vorentscheidung zum Status von Qualia (inexistent, marginal, identisch mit Physikalischem etc.) gerecht werden zu können, um so dem hard problem und dessen Explanandum ausweichen zu dürfen. So kann bspw. Churchland, aufgrund eines derartigen Qualia-Begriffs behaupten, Qualia seien ein »idiosynkratisches«14 und »marginales« ${ }^{15}$ Phänomen und folglich für eine Theorie des Geistes irrelevant. ${ }^{16}$ Wie Dennett beruft auch er sich auf jene angeblich notwendige

11 Dennett, Daniel C.: »Quining Qualia«, in: Philosophy of mind. Classical and contemporary readings, Chalmers, David J. [Hrsg.], New York: Oxford University Press [zuerst 1988]/2002, S. 227 .

12 So z.B. die repräsentationalistische Transformation von Farberleben bzw. Farb-Qualia, die in der externalistischen Version des Repräsentationalismus mit nicht-phänomenalen Explananda, nämlich den Oberflächeneigenschaften von Gegenständen, identifiziert werden. Vgl. dazu z.B. Tye, Michael: »Representationalist Theories of Consciousness«, in: The Oxford Handbook of Philosophy of Mind, McLaughlin, Brian P. / Beckermann, Ansgar / Walter, Sven [Hrsg.], Oxford: Oxford University Press 2009, S. 256. Vgl. auch Harman, Gilbert: »The intrinsic quality of experience«, in: Philosophy of mind. Critical concepts in philosophy. Volume 4: Consciousness, Crawford, Sean [Hrsg.], London: Routledge \& Kegan Paul [zuerst 199o]/2011, S. 110.

13 Vgl. Walter, Sven: »Allgemeine Einleitung: Phänomenales Bewusstsein: Unlösbares Mysterium oder seriöses wissenschaftliches Problem?«, in: Qualia. Ausgewählte Beiträge, Heckmann, Heinz-Dieter / Walter, Sven [Hrsg.], Paderborn: Mentis 2oo1, S. 56.

14 Churchland, Paul M.: »The Rediscovery of Light«, The Journal of Philosophy, [1996] Vol. 93, No. 5 , S. 223 .

15 Ebd.

16 Vgl. a.a.O., S. 223-224 und 228. Oder man transformiert den Qualia-Begriff, indem man behauptet, dass Phänomenales >nurく in einer >Fähigkeit<, in indexikalischen oder 
Qualia-Eigenschaft der »Introspektion $«{ }^{17}$ die wohl ihre $» S \operatorname{Sch} r$ llen $«(» q u i r k s \ll)^{18}$ hätten, aber von denen wir uns nicht »verführen «19 lassen sollen. Ebenfalls analog zu Dennett zieht Churchland zweifelhafte Aspekte einer besonders engen Qualia-Konzeption in Erwägung, um sich dann auf Beispielfälle zu beschränken, die nicht einmal die einfachen und herkömmlichen Standardfälle phänomenaler Bewusstseinszustände der sinnlichen Exterozeption in Erwägung ziehen, sondern nur Fälle, wie z.B. Tinnitus, innere Nachbilder oder Blasendruck, die seiner Introspektivitäts-Konzeption von »inneren Qualia « ${ }^{20}$ entsprechen. ${ }^{21}$ Die Relevanz von Qualia versucht er dann zu entkräften, indem er behauptet, sie bestünden ausschliesslich in einer epistemischen Modalität der »Introspektion «, ${ }^{22}$ die durch andere epistemische Modalitäten vollständig ersetzbar sei: »One can tell by introspection that one's own bladder is full, but an ultrasound image will tell anyone the same thing ${ }^{2}{ }^{23}$ Indem also Churchland eine volle Blase tatsächlich mit dem Erleben der Empfindung einer vollen Blase identifiziert, versucht er, neben der Limitierung von Qualia auf >Introspektion<, zusätzlich dem Explanandum phänomenaler Bewusstseinszustände auszuweichen. ${ }^{24}$

Nicht zuletzt versuchen auch nicht-eliminativistische Positionen, wie die von Dretske, Lycan oder Tye, auf der Basis einer phänomenal inadäquaten Vorentscheidung über die >wirkliche< Beschaffenheit von Qualia, diese bspw. auf externe ${ }^{25}$ Eigenschaften oder auf nicht-phänomenale Eigenschaften

phänomenalen Begriffen bestünde, womit man dann versucht, den epistemischen Wert des Erlebens anzuzweifeln um damit ebenfalls dem hard problem ausweichen zu können. Zur Interpretation von Erleben als >Fähigkeit`vgl. Lycan, William G. [Hrsg.]: Mind and cognition. An anthology, Malden, Massachusetts: Blackwell 1999, S. 459. Zu indexikalischen bzw. phänomenalen Begriffen vgl. Pauen, Michael / Schütte, Michael / Staudacher, Alexander [Hrsg.]: Begriff, Erklärung, Bewusstsein. Neue Beiträge zum QualiaProblem, Paderborn: Mentis 2007, S. 16-19 und 22. Zur Kritik am epistemischen Wert des Erlebens vgl. Metzinger, Thomas: Subjekt und Selbstmodell. Die Perspektivität phänomenalen Bewusstseins vor dem Hintergrund einer naturalistischen Theorie mentaler Repräsentation, Paderborn: Mentis 1999, S. 41.

17 Churchland, Paul M.: »The Rediscovery of Light«, The Journal of Philosophy, [1996] Vol. 93, No. 5 , S. 221, 226 und 227.

18 A.a.O., S. 221.

19 A.a.O., S. 226.

20 Ebd.

21 Vgl. dazu a.a.O., S. 226-227.

22 A.a.O., S. 221, 226 und 227.

23 A.a.O., S. 227.

24 Vgl. dazu ebd.

25 Vgl. z.B. Dretske, Fred: »Woher wissen Sie, dass Sie kein Zombie sind? «, in: Den eigenen Geist kennen. Selbstwissen, privilegierter Zugang und Autorität der ersten Person, Newen, Albert / Vosgerau, Gottfried [Hrsg.], Paderborn: Mentis [zuerst 2003, engl.]/2005, S. 102. 
intentionaler Repräsentationsgehalte ${ }^{26}$ zu reduzieren und in der Folge, Qualia subtil zu eliminieren. ${ }^{27}$ Das Vorgehen derartiger Qualiareduktions- bzw. QualiaEliminationsversuche vollzieht sich grob skizziert folgendermassen:

\section{A.) Variante von Positionen mit phänomenalem Antirealismus:}

1. Das reichhaltige, komplexe, heterogene und dynamische ${ }^{28}$ Spektrum phänomenalen Erlebens wird in einen, der Elimination dienlichen (besonders) engen, homogenen und statischen `Qualia<-Begriff transformiert (bzw. damit identifiziert).

2. Dieser `Qualia<-Begriff scheitert dann, freilich zwingend, am Desiderat phänomenaler Adäquatheit, was für seine Widerlegung aber hilfreich sein wird.

3. Im nächsten Argumentationsschritt werden dann die problematischen Aspekte dieser engen >Qualia<-Konzeption widerlegt. Infolge der phänomenalen Inadäquatheit einer solchen >Qualia<-Konzeption können die mit ihr verbundenen, vermeintlichen Qualia-Eigenschaften auch relativ leicht widerlegt werden. ${ }^{29}$

4. Auf der Grundlage der Widerlegung dieses verengten bzw. phänomenal inadäquaten \Qualia<-Begriffs wird dann gefolgert, dass phänomenales Erleben, egal wie es konzipiert sein mag, insgesamt ${ }^{30}$ nicht >existiert< und/oder das Explanandum wird wie folgt transformiert:

Vgl. ebenfalls Tye, Michael: »Qualia«, in: The Stanford Encyclopedia of Philosophy, 2007, Online: http://plato.stanford.edu/archives/fall2007/entries/qualia/ [zuletzt geprüft am 25.09.2018], Abschnitt 7.0.

26 Vgl. z.B. Lycan, William G.: Consciousness, Cambridge, Massachusetts: MIT Press 1987. Vgl. dazu auch Tye, Michael: »Representationalist Theories of Consciousness«, in: The Oxford Handbook of Philosophy of Mind, McLaughlin, Brian P. / Beckermann, Ansgar / Walter, Sven [Hrsg.], Oxford: Oxford University Press 2009, S. 262.

27 Vgl. dazu Tye, Michael: »Qualia«, in: The Stanford Encyclopedia of Philosophy, 2007, Online: http://plato.stanford.edu/archives/fall2007/entries/qualia/ [zuletzt geprüft am 25.09.2018], Abschnitt 6.o.

28 Der Begriff eines >dynamischen Spektrums< soll auf den diachronen Verlaufscharakter und auf die sich durch somatisch-emotive, kognitiv-emotive Prozesse ständig ändernden Varianten phänomenalen Erlebens hinweisen.

29 So z.B. die angebliche Umittelbarkeit, diachrone Infallibilität und Unveränderlichkeit des Erlebens von Kaffeegeschmack. Vgl.dazu Dennett, Daniel C.: »Quining Qualia«, in: Philosophy of mind. Classical and contemporary readings, Chalmers, David J. [Hrsg.], New York: Oxford University Press [zuerst 1988]/2002, S. 231-236. 


\section{B.) Variante von Positionen mit phänomenaler Indifferenz:}

1. Phänomenale Explananda werden auf eine, der Explizierbarkeit (vermeintlich $)^{31}$ dienlichen Weise, in nicht-phänomenale Explananda transformiert.

2. Aufgrund dieser Transformation werden Explananda des hard problem durch Explananda des easy problem ausgetauscht. So verschiebt sich der ursprüngliche Fokus weg von Explananda phänomenalen Erlebens (welche das hard problem konstituieren) auf Explananda, wie beispielsweise repräsentationale Gehalte ${ }^{32}$, metarepräsentationale Gehalte, ${ }^{33}$ Farboberflächeneigenschaften, ${ }^{34}$ neurophysiologische Prozesse ${ }^{35}$ oder funktionale bzw. kausale Rollen, die das easy problem auszeichnen. ${ }^{36}$

3. Infolge der Verschiebung der Aufmerksamkeit auf die einfacheren Explananda des easy problem, werden auf Fragen zum hard problem (detailliert ausgearbeitete) Antworten auf Explananda des easy problem angeboten. ${ }^{37}$

$31 \quad$ Freilich ist dies nur scheinbar für die Explizierbarkeit des Phänomenalen dienlich, weil sich aufgrund der Transformation der Explananda die Erklärungen auf etwas NichtPhänomenales beziehen.

32 Vgl. z.B. Lycan, William G.: Consciousness, Cambridge, Massachusetts: MIT Press 1987. Vgl. ebenfalls Tye, Michael: »Representationalist Theories of Consciousness«, in: The Oxford Handbook of Philosophy of Mind, McLaughlin, Brian P. / Beckermann, Ansgar / Walter, Sven [Hrsg.], Oxford: Oxford University Press 2009, S. 262.

33 Vgl. dazu z.B. Carruthers, Peter: »Higher-Order Theories of Consciousness«, in: The Stanford Encyclopedia of Philosophy, 2016, Online: https://plato.stanford.edu/archives/ fall2016/entries/consciousness-higher/ [zuletzt geprüft am 25.07.2017], Abschnitt 4.o. Vgl. auch Metzinger, Thomas [Hrsg.]: Grundkurs Philosophie des Geistes. Band 3: Intentionalität und mentale Repräsentation, Paderborn: Mentis 2007, S. 26.

34 Vgl. z.B. Dretske, Fred: »Woher wissen Sie, dass Sie kein Zombie sind? «, in: Den eigenen Geist kennen. Selbstwissen, privilegierter Zugang und Autorität der ersten Person, Newen, Albert / Vosgerau, Gottfried [Hrsg.], Paderborn: Mentis [zuerst 2003, engl.]/2005, S. 102. Vgl. ebenfalls Tye, Michael: »Qualia«, in: The Stanford Encyclopedia of Philosophy, 2007, Online: http://plato.stanford.edu/archives/fall2007/entries/qualia/ [zuletzt geprüft am 25.09.2018], Abschnitt 7.0.

35 So behauptet z.B. Papineau:»[...] the material property [...] is the conscious property. [...] feeling a pain is having your C-fibres firing. [...] The >two< states are the same, and that's it«. Papineau, David: »Mind the Gap«, Philosophical Perspectives, [1998] Vol. 12: Language, Mind, and Ontology, S. 379 .

36 Vgl. z.B. Lewis, David: »Psychophysical and theoretical identifications«, Australasian Journal of Philosophy, [1972] Vol. 5o, No. 3, S. 249-258. Vgl. dazu auch Armstrong, David M.: The nature of mind, Brighton, Sussex: The Harvester Press 1980.

37 Vgl. z.B. Lycan, William G.: Consciousness, Cambridge, Massachusetts: MIT Press 1987. Vgl. auch Tye, Michael: »Representationalist Theories of Consciousness «, in: The Oxford Handbook of Philosophy of Mind, McLaughlin, Brian P. / Beckermann, Ansgar / Walter, Sven [Hrsg.], Oxford: Oxford University Press 2009, S. 262. 
4. Dies (Nr. 3) bedeutet aber, dass für die typischen Explananda des hard problem mehr oder weniger irrelevante Explanantia ${ }^{38}$ ausgearbeitet werden, welche ganz im Sinne des »explanatory gap «39 keine hinreichende Erklärung darzustellen vermögen.

5. Auf diese Weise wird schliesslich das hard in ein easy problem umgedeutet ${ }^{40}$ - das gleicht aber eher einem Wegdefinieren, als einer Lösung des hard problem. Dass dabei, im Hinblick auf das hard problem nichts gewonnen wird, soll hier mit Chalmers nochmals auf den Punkt gebracht werden: »One might as well define >world peace< as a >ham sandwich $<$. Achieving world peace becomes much easier, but it is a hollow achievement « ${ }^{41}$

\subsection{Verschiebung der Patt-Situation}

Wenn Qualia nicht wie behauptet ein bloss »marginales «, ${ }^{42}$ sondern wie ich in meiner Qualia-Revision dargelegt habe, ein ubiquitäres Bewusstseinsphänomen darstellen, dann verschiebt sich die Beweislast im Patt zwischen reduktionsoptimistischen und reduktionskritischen Positionen: Ansätze mit phänomenaler Indifferenz oder phänomenalem Antirealismus ${ }^{43}$ müssen umso dringlicher begründen, warum für eine Theorie des Geistes, Phänomenalität keine Rolle spielen soll. Wenn Phänomenalität aber nicht nur ubiquitär ist, sondern unentwirrbar mit Bewusstsein und kognitiven Prozessen zusammenhängt, mit welchem Grund kann es dann bei einer Theorie des Geistes, die wohl schwer Bewusstsein und Kognition ausklammern kann, ausser Acht gelassen, vernachlässigt oder gar negiert werden?

Wenn reduktionsoptimistische Ansätze auf das hier angesprochene Problem der Ubiquität von Qualia und phänomenalem Erleben sich wiederum auf ontologische Einfachheit oder einfachere Explizierbarkeit zurückziehen, so ist diese letzte Bastion noch angreifbarer geworden: denn wenn Phänomenalität

\footnotetext{
$38 \quad$ (Explanantia ist der Plural von Explanans)

39 Levine, Joseph: »Materialism and Qualia: The Explanatory Gap«, Pacific Philosophical Quarterly, [1983] Vol. 64, No. 4, S. 357.

40 Vgl. dazu auch Jung, Matthias / Heilinger, Jan-Christoph [Hrsg.]: Funktionen des Erlebens. Neue Perspektiven des qualitativen Bewusstseins. Band 5: Humanprojekt, Berlin: De Gruyter 2009, S. 7 .

41 Chalmers, David J.: The conscious mind. In search of a fundamental theory, New York: Oxford University Press 1996, S. 105.

42 Churchland, Paul M.: »The Rediscovery of Light«, The Journal of Philosophy, [1996] Vol. 93, No. 5 , S. 223 .

43 Vgl. dazu Abschnitte 5.6, 7.1 und 7.2
} 
ein ubiquitäres Bewusstseinsphänomen ist, so ist ihr Scheitern bezüglich der für eine erfolgreiche Reduktion geforderten »Rettung der Phänomene « ${ }^{44}$ noch schwerwiegender als beim herkömmlichen Qualia-Begriff der Standardbeispiele >Schmerz< bzw. monosensualer `Sinneserlebnisse $<$. Wenn Qualia tatsächlich, wie auf der Grundlage von verengten Qualia-Begriffen behauptet wird, eigentümliche Eigenschaften, wie die einer isolierten >Homogenität<, blossen >Innerlichkeit<, >Introspektivität< etc. hätten, und deshalb aufgrund einer damit verbundenen Marginalität nur ein vernachlässigbares Phänomen darstellen würden, wäre es schon so, dass 〉Qualia-Freaks< aufzeigen müssten, warum sie trotzdem als Explanandum von Relevanz sind.

Mit dem Nachweis der Ubiquität von phänomenalem Erleben, geht die Beweislast aber zurück an die Qualia-Skeptiker und noch mehr an Eliminativisten. Sie müssen ausweisen, warum diejenige Eigenschaft, die infolge ihrer Ubiquität und ihrer Bewusstseinsverwobenheit bewusste Organismen auszeichnet, kein Explanandum sein soll. Dieser Ausweis muss ohne Ausflüchte auf andere Problemoptiken (wie bspw. die oben erwähnte Achse kausaltheoretischer Überlegungen), ${ }^{45}$ die vom hard problem ablenken sollen, gebracht werden. Das heisst, Reduktionsoptimisten müssen aufzeigen, auf welche Weise phänomenales Erleben auf Dritte-Person-Eigenschaften erfolgreich, sprich: unter Berücksichtigung der geforderten »Rettung der Phänomene « ${ }^{46}$ reduzierbar ist, und warum sich das hard problem vom easy problem nicht unterscheiden soll. Auf der Grundlage eines phänomenal adäquaten Qualia-Begriffs lässt sich ebenfalls aufzeigen, dass sich phänomenales Erleben nicht in die angeblich marginalen epistemischen Bereiche der »Innerlichkeit « ${ }^{47}$ und $»$ Introspektion $\ll^{48}$ abschieben lässt, sondern auch in Form gewöhnlicher, (oft

44 Schildknecht, Christiane: »Der Dualismus und die Rettung der Phänomene«, in: Homo Sapiens und Homo Faber. Epistemische und technische Rationalität in Antike und Gegenwart. Festschrift für Jürgen Mittelstrass, Wolters, Gereon / Mittelstrass, Jürgen [Hrsg.], Berlin: W. de Gruyter 2005, S. 225.

45 Vgl. dazu Abschnitt 5.

46 Ebd. Vgl. dazu auch Nagel, Thomas: »Consciousness and Objective Reality«, in: The mindbody problem. A guide to the current debate, Warner, Richard / Szubka, Tadeusz [Hrsg.], Cambridge, Massachusetts: Blackwell 1994, S. 67.

47 Metzinger, Thomas: Subjekt und Selbstmodell. Die Perspektivität phänomenalen Bewusstseins vor dem Hintergrund einer naturalistischen Theorie mentaler Repräsentation, Paderborn: Mentis 1999, S. 37. Vgl. ebenfalls Dennett, Daniel C.: »Quining Qualia«, in: Philosophy of mind. Classical and contemporary readings, Chalmers, David J. [Hrsg.], New York: Oxford University Press [zuerst 1988]/2002, S. 229.

48 Metzinger, Thomas: Subjekt und Selbstmodell. Die Perspektivität phänomenalen Bewusstseins vor dem Hintergrund einer naturalistischen Theorie mentaler Repräsentation, Paderborn: Mentis 1999, S. 38. 
veridischer) exterozeptiver Wahrnehmungen zu den wichtigsten Merkmalen und Fähigkeiten unseres Geistes gehört. ${ }^{49}$

Die Beweislast fällt folglich denjenigen Positionen mit physikalistischmonistischer Ontologie zu, die behaupten, dass Qualia nur im Bereich »Inner-

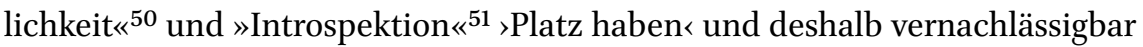
seien. Derartige mit einem verengten Qualia-Begriff zusammenhängenden Verdrängungen des Explanandums in eine vermeintlich zu vernachlässigenden Domäne lassen sich nur temporär aufrecht erhalten. Denn sie werden spätestens dann unbefriedigend, wenn die Aufmerksamkeit - anstatt dass sie bei den Vorentscheidungen über Qualia bequemen verweilt - auf die von jeder Theorie geforderte Rettung der Phänomene zurückfällt. Ist diese Verdrängung der Anerkennung des Desiderates für jede erfolgreiche Reduktion gewichen, so muss die Aufmerksamkeit leider auf ein unbequemes, da schwer zu erklärendes - aber unvernachlässigbares, weil ubiquitäres Bewusstseinsphänomen fallen:

By removing these troublesome properties from the world and locking them up in the Mind the world is opened up for scientific, naturalistic explanation. But this solution is only temporary. For the scientific picture of the world is incomplete as long as it does not encompass its creators. ${ }^{52}$

\subsubsection{Aufwertung von Theorien des Geistes, die Erleben einbeziehen}

Mit dieser Qualia-Revision wurde aufgezeigt, dass Erlebnisqualitäten ein ubiquitäres Bewusstseinsphänomen darstellen und sich daher eine Ausklammerung dieses Kern-Explanandums als noch viel problematischer ausweist, als sich dies schon bei einem herkömmlichen Qualia-Begriff zeigt (vgl. Kapitel 9). Positionen wie z.B. der Eliminativismus, identitätstheoretische oder funktionalistisch-reduktionistische Ansätze, vermögen aufgrund ihrer Ausklammerung von bewusstem Erleben und der daraus folgenden phänomenalen

49 Vgl. dazu auch Langsam, Harold L.: »Experiences, Thoughts, and Qualia«, Philosophical Studies, [2000] Vol. 99, No. 3, S. 269. Vgl. dazu auch Beaton, Michael: »Qualia and Introspection«, Journal of Consciousness Studies, [2009] Vol. 16, No. 5, S. 105. Vgl. dazu ebenfalls Abschnitte 8.2, 9.2 und 9.3.

$50 \quad$ Dennett, Daniel C.: »Quining Qualia «, in: Philosophy of mind. Classical and contemporary readings, Chalmers, David J. [Hrsg.], New York: Oxford University Press [zuerst 1988]/2002, S. 229 .

51 Metzinger, Thomas: Subjekt und Selbstmodell. Die Perspektivität phänomenalen Bewusstseins vor dem Hintergrund einer naturalistischen Theorie mentaler Repräsentation, Paderborn: Mentis 1999, S. 38 .

$5^{2}$ Stubenberg, Leopold: Consciousness and qualia, Amsterdam: John Benjamins 1998, S. 3 [Hervorhebung E. E.]. 
Inadäquatheit, umso stärker mit ihren Explanantia keinen sinnvollen Bezug zum Explanandum zu gewährleisten. Auch der Versuch, Bewusstsein bei der (vermeintlichen) Reduktion von phänomenalen in intentionale Explananda auszuklammern, kann aufgrund der in Abschnitt 8.2.5 ausgewiesenen phänomenalen Aspekte des Intentionalen nicht gelingen.

Der Nachweis von Qualia als ubiquitäres Bewusstseinsphänomen zieht konsequenterweise eine Aufwertung von Theorien des Geistes nach sich, die Erleben und Bewusstsein in ihre Theorien einzubeziehen vermögen, d.h., das Explanandum des hard problem nicht ausklammern und deshalb in ihren Erklärungsansätzen auch nicht das Prinzip der Rettung der Phänomene verletzen. Letzteres bedeutet eben, dass eine Theorie des Geistes nicht bloss der Forderung nach physikalischer, sondern auch derjenigen nach phänomenaler Adäquatheit standhalten muss. Theorien, die diesen Anforderung nachkommen, sind z.B. Versionen des neutralen Monismus, der Eigenschaftsdualismus, des nicht-reduktiven Funktionalismus bzw. schwachen Repräsentationalismus, des Protopanpsychismus, Panpsychismus, Mysterianismus etc. und/oder Theorien, die nach Chalmers' Systematik, so genannte »Typ-C views« über bewusstes Erleben darstellen.53 Auf solche Varianten von Theorien des Geistes, die nicht nur dem Desiderat physikalischer Adäquatheit, sondern auch dem der phänomenalen Adäquatheit nachzukommen versuchen, gehe ich kurz in Abschnitt 10.3 ein. Positionen, die Phänomenales wenigstens als ein Explanandum anerkennen, ohne es zu transformieren bzw. subtil zu eliminieren, sind etwa der starke Emergentismus, Epiphänomenalismus oder der anomale Monismus.

\subsubsection{Aporie für Theorien des Geistes, die Erleben systematisch ausklammern}

Gegenüber einer Aufwertung von Theorien des Geistes, die das Explanandum $>$ Erleben< hinreichend beachten, führt bspw. die auf Fallibilitätsargumenten ${ }^{54}$

53 Typ-C Positionen bestreiten die logische Supervenienz von phänomenalen Eigenschaften über physikalischen Eigenschaften und sind gegen materialistisch-reduktionistische Ansichten über Phänomenales. Eine Typ-C Position »[...] takes phenomenal or protophenomenal properties as fundamental $[. .$.$] «. Chalmers, David J.: The conscious$ mind. In search of a fundamental theory, New York: Oxford University Press 1996, S. 166. Typ-C Positionen können eigenschaftsdualistisch sein (z.B. Campbell, Chalmers, Honderich, Jackson, H. Robinson, W. Robinson, Sprigge, vgl. ebd.) oder Versionen von neutralen Monismen vertreten (z.B. Feigl, Lockwood, E. Mach, Maxwell, Russell, M. Velmans, W. James). Vgl. ebd.

54 Vgl. dazu Willaschek, Marcus: »Wahrnehmung VII (Analytische Philosophie)«, in: Historisches Wörterbuch der Philosophie. Band 12: W-Z, Ritter, Joachim / Kranz, Margarita [Hrsg.], Darmstadt: Wissenschaftliche Buchgesellschaft 1971-2007, S. 231-232. Vgl. auch 
basierende Behauptung von Qualia-Skeptikern oder Eliminativisten, dass es >in Wirklichkeit « vielleicht gar kein bewusstes Erleben "gibt « ${ }^{55}$ in eine Aporie. Denn selbst dann, wenn man ihre Behauptung lediglich als Arbeitshypothese akzeptieren würde, dann wären Philosophen oder Neurowissenschafter mindestens bezüglich der Beurteilung von Phänomenalem - in der epistemisch aussichtslosen Lage eines Zombies und könnten das im Diskurs um das hard problem umstrittene Explanandum gar nicht verstehen. Infolgedessen wüssten sie auch nicht, was im hard problem umstritten ist bzw. welchen Phänomenen sie eigentlich die Existenz absprechen. Eine damit verbundene, wohl letzte Ausweichstrategie von Bewusstseins- und Qualia-SkeptikerInnen oder Eliminativisten läge noch darin, zu behaupten, dass wir zwar keine Zombies sind, es aber dennoch nur so »scheine ${ }^{56}$, als ob es phänomenales Erleben gäbe; und wir deshalb, auch wenn Bewusstsein nicht >wirklichく sei, es mit unserer »Alltagsintuition ${ }^{57}$ schon verstehen können. Diese Argumentation führt aber insofern in eine Aporie, als sie Qualia-SkeptikerInnen, die sich auf die angebliche `Scheinbarkeit $>$ von Qualia berufen, vor die unattraktive Wahl folgender zwei Möglichkeiten stellt:

A.) Beruft man sich auf eine solche Argumentation, die ich hier als iit»seems « ${ }^{58}$ Argument $<$ bezeichne (es >scheint nur so, als ob es phänomenales Erleben gäbe), so tangiert dieses Argument den bestrittenen Erlebnischarakter gar nicht: ob es sich bei bewusstem Erleben um ein Erleben handelt, welches >eigentlich $<$ eine >Täuschung $<$ ist, ist im Hinblick auf das diskutierte Phänomen, dass erlebt wird, gar nicht relevant (vgl. dazu auch Abschnitte 8.1 und 9.2). Das Explanandum >Erleben<, ob es nun veridisch ist oder nicht, kann mit der skeptizistischsten Deutungsvariante allenfalls als ein $>$ Erleben-einer-Bewusstseins-und-Welt-Illusion< gedeutet werden - jedoch nicht durch einen prädizierenden Zauber, der

Dennett, Daniel C.: »Quining Qualia«, in: Philosophy of mind. Classical and contemporary readings, Chalmers, David J. [Hrsg.], New York: Oxford University Press [zuerst 1988]/2002, S. 238-240.

55 A.a.O., S. 244.

56 Dennett, Daniel C.: »The Mystery of David Chalmers«, Journal of Consciousness Studies, [2012] Vol. 19, No. 1-2, S. 88.

57 (bzw. »folk convictions«) Dennett, Daniel C.: »Why and How Does Consciousness Seem the Way it Seems?«, 2015, Online: http://open-mind.net/papers/why-and-how-doesconsciousness-seem-the-way-it-seems [zuletzt geprüft am 24.09.2018], S. 4.

$5^{8}$ Dennett, Daniel C.: »The Mystery of David Chalmers«, Journal of Consciousness Studies, [2012] Vol. 19, No. 1-2, S. 88. 
lautet: $>$ Erleben ist illusorisch $<$, in ein $>$ zombiartiges Nicht-Erleben $<$ transmutieren. ${ }^{59}$

B.) Falls jemand, trotz den Problemen von Option A.) an jener Umdeutung von Erleben in ein >zombieartiges Nicht-Erleben< festhält, führt dies $\mathrm{zu}$ weiteren, noch schwerwiegenderen Problemen: Macht eine Anhängerin des Fallibilitätsargumentes den eliminativen Aspekt so stark, dass damit die Behauptung einhergeht, dass es >in Wirklichkeit< weder das Erleben einer >Täuschung< bzw. einer >Bewusstseins-Illusion< noch sonst irgendeine Form von Erleben >gibt<, dann erweist sich diese letzte Bastion des Eliminativismus als Fallgrube mit diversen Widersprüchen: Wie könnten PhilosophInnen und NeurowissenschafterInnen überhaupt auf Fragen über phänomenales Erleben kommen und sich Gedanken darüber machen, wenn sie nie irgendetwas, d.h. nicht einmal die angebliche >Täuschung<, dass sie erlebend sind, erlebt haben? Wie könnte eine Eliminativistin, wenn sie nie etwas erlebt hat, auch nur ansatzweise verstehen, von welchen Phänomenen sie redet, wenn sie behauptet, Euphoriegefühle von Menschen seien nur eine >Täuschung<, wirklich seien nur die mit dieser Illusion korrelierenden neurophysiologischen Vorgänge im Gehirn. ${ }^{60}$ Ja wie könnte sie, die nie etwas erlebt hat, überhaupt widerspruchsfrei behaupten, dass bestimmte neuronale Prozesse uns das Erleben dieser Euphorie nur >vorgaukeln<, wenn es gar >nichts $<$ gibt, das >vorgegaukelt< werden kann? Aus diesem Widerspruch kann man sich nur noch mit der oben erwähnten Option A, mit dem >it»seems «61-Argument< retten, doch letzteres tangiert den bestrittenen Erlebnischarakter leider gar nicht, weil die Nicht-Veridität des Erlebens, erlebende Subjekte nicht in Zombies zu verwandeln vermag.

59 Diesbezüglich lässt sich mit Ernst Mach die Frage stellen: »Was berechtigt uns aber, eine Tatsache der andern gegenüber für Wirklichkeit zu erklären und die anderen zum Schein herabzudrücken? Auch der wüsteste Traum ist eine Tatsache [...] «. Mach, Ernst: Die Analyse der Empfindungen und das Verhältnis des Physischen zum Psychischen, Saarbrücken: VDM Verlag Dr. Müller 20o6, S. 8. Zitiert nach: Kaiser-El-Safti, Margret: »Realität, psychische«, in: Historisches Wörterbuch der Philosophie. Band 8: R-Sc, Ritter, Joachim / Kranz, Margarita [Hrsg.], Darmstadt: Wissenschaftliche Buchgesellschaft 19712007, S. 205.

6o Vgl. dazu auch Vogelsang, Frank: Offene Wirklichkeit. Ansatz eines phänomenologischen Realismus nach Merleau-Ponty, Freiburg: Alber 2011, S. 420.

61 Dennett, Daniel C.: »The Mystery of David Chalmers«, Journal of Consciousness Studies, [2012] Vol. 19, No. 1-2, S. 88. 


\subsection{Ist Phänomenale Adäquatheit ohne Dualismus zu haben?}

\subsubsection{Qualia- und Materie-Begriff als revisionsbedürftig?}

Wir haben nun die Wahl, den Begriff >Qualia<, wenn er weiterhin bloss auf eine marginale und seltsame Form des Erlebens referieren soll, im Diskurs um das hard problem abzuschaffen. Dann müssten wir aber, um dem Desiderat phänomenaler Adäquatheit gerecht zu werden, im Zusammenhang mit dem hard problem vom $>$ Problem des Bewusstseins $<$ bzw. vom $>$ Problem des Erlebens überhaupt « sprechen. So betrachtet, wäre eine >Eliminierung « von Qualia, im oben kritisierten, verengten Sinne sogar die Voraussetzung dafür, zu erkennen, dass das hard problem noch viel schwerwiegender ist, als bisher angenommen.

Eine andere Möglichkeit, besteht freilich darin, den Qualia-Begriff so zu erweitern, dass er dem Desiderat phänomenaler Adäquatheit standhält. Im Hinblick auf das hard problem spielt es letztlich keine Rolle, mit welchen Begriffen wir auf das Explanandum bzw. auf das Phänomen, dass wir erleben (selbst wenn es nur das Erleben einer >Täuschung〈, dass wir erleben wäre) referieren. Ungeachtet durch welche Problemachse ${ }^{62}$ wir dieses Phänomen auch betrachten und in welches theoretische System wir es einordnen - oder mit welchen Begrifflichkeiten wir seine Relevanz zu marginalisieren oder es Zwecks besserer Explizierbarkeit zu transformieren versuchen - lässt sich feststellen, dass aufgrund der Ubiquität phänomenalen Erlebens ein simples aber grundlegendes Problem schwerwiegender wird: Theorien des Geistes, die versuchen, phänomenale Explananda auf nicht-phänomenale Eigenschaften zu reduzieren, fehlt etwas. ${ }^{63}$

62 Im Hinblick auf die verschiedenen Problemachsen bzw. Problemoptiken, durch welche man das hard problem betrachten kann, lässt sich analog feststellen, dass unbeachtet, ob wir letzteres durch die ontologische, kausale, explanatorisch-reduktionstheoretische oder mereologische Achse der Problemoptik betrachten, sich die phänomenale Problemachse nicht aus dem Weg räumen lässt. Das heisst, dass sich das Explanandum des hard problem - phänomenales Erleben - nicht einfach aufgrund einer anderen Problemoptik aufzulösen beginnt.

63 Ähnlich formuliert es Chalmers im Hinblick auf aposteriorische Einwände gegen modale Argumente, wie >Bewusstsein < im Hinblick auf die primäre oder sekundäre Intension von Bewusstsein semantisch verstanden werden könnte: »[...] the relevant possible world clearly lacks something, whether or not we call it >consciousness ««. Chalmers, David J.: The conscious mind. In search of a fundamental theory, New York: Oxford University Press 1996, S. 134 [Hervorhebung im Original]. Oder mit den Worten Pitts: »Phenomenology is not describable in nonphenomenological terms; and different sorts of phenomenology are not describable in the terms of any other sort«. Pitt, David: »The Phenomenology of Cognition or what is it Like to Think that P? «, Philosophy and Phenomenological Research, [2004] Vol. 69, No. 1, S. 31-32. 
Die zentrale Intuition eines reduktiven Materialismus' besteht nun aber nichtsdestotrotz darin, dass die Beziehung zwischen dem >Mentalen $<$ und dem >Physischen< als asymmetrische Beziehung gesehen wird: »Das Physische ist in wesentlicher Hinsicht fundamentaler als das Mentale. Das Mentale hängt demnach vom Physischen $a b \ll \cdot{ }^{64}$ Daher könnte der Grund für die Idee, dass sich Phänomenales ohne jegliche Probleme auf Nicht-Phänomenales reduzieren lasse, auch darin liegen, dass die reduktionistische Intuition besagt, dass es möglich sein muss, Materie >unabhängig`von Bewusstsein zu >erkennen` und anschliessend zu konzeptualisieren, obwohl wir erst auf der Grundlage von Bewusstsein, bzw. bewusster Kognition, Materie erkennen und dann überhaupt auf so etwas, wie einen Materiebegriff kommen können. So können wir mit Huxley konstatieren, dass es unmöglich ist, einen Materie-Begriff so zu konstruieren, dass diese Konstruktion je einmal als unabhängig und isoliert von unserem Bewusstsein gelten könnte: »[...] I am utterly incapable of conceiving the existence of matter if there is no mind in which to picture that existence $[\ldots] \ll .65$

Dennoch geht man bei der Ansicht, dass das, was wir unter >Materie< verstehen, irgendwie >realer ist, als das was wir erleben, davon aus, dass >materielle< Eigenschaften eines Hirns irgendwie >realer` sein müssen als $>$ mentale< Eigenschaften. ${ }^{66}$ Und es liegt wohl an dieser Konzeption, dass man hofft, Qualia ohne Verlust und ohne Probleme auf Gegenständliches reduzieren zu können. Dass man phänomenale Eigenschaften nicht im Sinne des Externalismus als physikalische Eigenschaften von Gegenständen >vor< unseren Sinnesorganen findet, wird jedoch, wie ich in Abschnitt 7.2.3 aufgezeigt habe, durch wahrnehmungstheoretische Überlegungen evident: Die Strukturen unserer subjektiven Farbwahrnehmung entsprechen z.B. nicht den Strukturen derjenigen physikalischen Eigenschaften, die mit phänomenalem Erleben zusammenhängen. ${ }^{67}$ Und weil man phänomenale Bewusstseinszustände weder >in< Schallwellen, Oberflächeneigenschaften, Molekülen von

64 Staudacher, Alexander: Phänomenales Bewusstsein als Problem für den Materialismus, Berlin: De Gruyter 2002, S. 16 [Hervorhebung im Original].

65 Huxley, Thomas H.: »On the hypothesis that animals are automata, and its history«, in: Collected Essays, Volume I, Method and Results, Huxley, Thomas H. [Hrsg.], London: Macmillan [zuerst 1874]/1893, S. 245 .

66 Vgl. dazu Staudacher, Alexander: Phänomenales Bewusstsein als Problem für den Materialismus, Berlin: De Gruyter 2002, S. 16-17.

67 Beispiele von diesen Strukturdifferenzen, insbesondere im Hinblick auf visuelle Wahrnehmungen, finden sich vor allem bei Lanz, vlg. Lanz, Peter: Das phänomenale Bewusstsein. Eine Verteidigung, Frankfurt am Main: Klostermann 1996, S. 123, 126 und 139. Vgl. dazu Nagel, Thomas: Was bedeutet das alles? Eine ganz kurze Einführung in die Philosophie, Stuttgart: Reclam 2012, S. 33-34. 
Geschmacks- oder Duftstoffen etc. noch >in neurophysiologischen Korrelaten $^{68}$ findet, folgern reduktionistische Positionen, dass sich für sie auch »kein Platz«69 innerhalb eines wissenschaftlichen Weltbildes finden lässt. Der Umstand, dass sich Phänomenalität nicht auf Dritte-Person-Eigenschaften reduzieren lässt bzw. Erleben sich nicht >inく Objekten finden lässt, bedeutet aber nicht, dass phänomenale Adäquatheit per se einem wissenschaftlichen Weltbild >widersprechen muss, sondern nur, dass Bewusstsein selbst kein beobachtbares Objekt ist: »[...] the property of consciousness itself [...] is not an observable or perceptible property of the brain «. ${ }^{70}$

Einen Ausweg aus der daraus entstehenden, schier unlösbar anmutenden Anforderung, sowohl dem Desiderat phänomenaler Adäquatheit stand zu halten, als auch die Probleme eines ontologischen Dualismus zu vermeiden, könnten Positionen, wie die des dualen Aspekt-Monismus (dual-aspect monism $),{ }^{71}$ des neutralen Monismus, ${ }^{72}$ des reflexiven Monismus, ${ }^{73}$ der neutralmonistischen Variante des Panpsychismus ${ }^{74}$ etc. darstellen. Denn solche

68 Darauf weist bspw. das oben angesprochene, ziemlich drastische Gedankenexperiment von Thomas Nagel, in welchem er darlegt, dass ein Wissenschaftler, wenn er an einem Hirn leckte von jemandem die oder der gerade Schokolade isst, wohl nicht den Geschmack von Schokolade erleben würde. Vgl. dazu ebd.

69 Beckermann, Ansgar: Analytische Einführung in die Philosophie des Geistes, Berlin / New York: Walter de Gruyter Verlag 2008, S. $45^{6}$.

70 McGinn, Colin: »Can We Solve the Mind-Body Problem?«, Mind (New Series), [1989] Vol. 98, No. 391, S. 356-357.

71 Für den dualen Aspekt-Monismus sind Mentales und Materielles zwar irreduzibel, aber beide Aspekte lassen sich auf eine, ihnen zugrunde liegende »neutrale Domäne« zurückführen. Vgl. Atmanspacher, Harald: »Dual-Aspect Monism à la Pauli and Jung«, Journal of Consciousness Studies, [2012] Vol. 19, No. 9-10, S. 97. Vgl. ebenfalls Atmanspacher, Harald: »Quantum Approaches to Consciousness«, in: The Stanford Encyclopedia of Philosophy, 2015, Online: http://plato.stanford.edu/archives/sum2015/entries/qt-consciousness [zuletzt geprüft am 28.o9.2018], Abschnitt 4.6.

72 Positionen des neutralen Monismus bzw. des reflexiven Monismus gehen, vereinfacht gesagt, davon aus, dass physikalische und mentale Eigenschaften auf ontologisch gewissermassen >neutralen< Eigenschaften gründen. Vgl. z.B. Alter, Torin / Nagasawa, Yujin: »What is Russellian Monism?«, Journal of Consciousness Studies, [2012] Vol. 19, No. 9-10, S. 78. dazu auch Vgl. Velmans, Max:»Reflexive Monism. Psychophysical Relations among Mind, Matter, and Consciousness «, Journal of Consciousness Studies, [2012] Vol. 19, No. 9-10, S. 143-165.

73 Vgl. ebd.

74 Der Panpsychismus kann jedoch auch in einer immaterialistisch-monistischen Variante und in einer Dual-Aspekt-Version auftreten. Vgl. dazu Weekes, Anderson: »The MindBody Problem and Whitehead's Non-reductive Monism«, Journal of Consciousness Studies, [2012] Vol. 19, No. 9-10, S. 49. Bezüglich der neutralmonistischen Variante des Panpsychismus (mit anderen Worten: die panpsychistische Variante des neutralen Monismus) vgl. Seager, William / Allen-Hermanson, Sean: »Panpsychism«, in: The Stanford 
Positionen sind einerseits nicht dualistisch, andererseits reduzieren sie nicht einfach, wie strikt monistische Positionen, aufgrund der Präferenz einer immaterialistischen bzw. materialistischen Ontologie, physikalische Phänomene auf mentale Eigenschaften bzw. mentale Phänomene auf physikalische Eigenschaften. ${ }^{75}$

Es ist also möglich, Bewusstsein und Physikalisches als irreduzibel zu konzeptualisieren und dennoch keinen ontologischen Dualismus vertreten zu müssen. Das cartesianische Erbe sorgt jedoch dafür, dass nicht wenige Philosophen glauben, sie müssten sich entscheiden, entweder »Materialisten « oder »Dualisten $\ll^{76}$ zu sein. Konsequent nicht-reduktive Positionen, d.h. solche, die von der Präferenz einer materialistischen oder immaterialistischen Ontologie absehen, gehen demgegenüber davon aus, dass es weder unabhängige physikalische noch unabhängige mentale Eigenschaften gibt, ${ }^{77}$ und dass die dem subjektiven Erleben bzw. den neuronalen Korrelaten zugrundeliegenden Eigenschaften etwas anders als nur >mentales< oder nur >physikalisches< im herkömmlichen Sinne sind:

[...] whatever the nature of mind might be, under respectively first- and thirdperson observation arrangements it must have the power to give rise to its

Encyclopedia of Philosophy, 2013, Online: http://plato.stanford.edu/archives/fall2013/ entries/panpsychism/ [zuletzt geprüft am 25.09.2018], Abschnitt 3.o.

75 So z.B. die immaterialistisch-monistische Variante des Panpsychismus, der Solipsismus oder Varianten des Idealismus, nach welchen der Geist die Grundlage der gesamten Realität bildet; oder die materialistisch-monistische Variante des Eliminativismus, nach welchem es nur relationale bzw. objektive Phänomene (wie z.B. Sprachverhalten, mit welchem ein Organismus von subjektivem Erleben spricht) >gibt<, aber keine mentale Phänomene des Erlebens. Zur strikt monistischen, immaterialistisch Variante des Panpsychismus vgl. Clifford, William: »On the Nature of Things-in-Themselves«, Mind, [1878] Vol. 3, No. 9, S. 57-67. Zum Idealismus Vgl. Guyer, Paul / Horstmann, Rolf-Peter: »Idealism«, in: The Stanford Encyclopedia of Philosophy, 2015, Online: http://plato.stanford.edu/archives/fall2015/entries/idealism/ [zuletzt geprüft am 26.o9.2016], Abschnitt 1.o. Zum strikt monistischen Materialismus des Eliminativismus vgl. z.B. Rorty, Richard: Philosophy and the mirror of nature, Oxford: Blackwell 1979, S. 22. Oder: Churchland, Paul M.: Matter and consciousness. A contemporary introduction to the philosophy of mind, Cambridge, Massachusetts: MIT Press 1988. Oder: Dennett, Daniel C.: »Quining Qualia«, in: Philosophy of mind. Classical and contemporary readings, Chalmers, David J. [Hrsg.], New York: Oxford University Press [zuerst 1988]/2002, S. 226-246.

76 Searle, John R.: The mystery of consciousness, New York: New York Review Books 1997, S. 195 .

77 So z.B. geht der neutrale Monismus davon aus, dass dem, was man im herkömmlichen Sinne unter >physikalisch bzw. unter >mentalく versteht, eine neutrale Eigenschaft zugrunde liegt. Vgl. dazu auch Alter, Torin / Nagasawa, Yujin: »What is Russellian Monism?«, Journal of Consciousness Studies, [2012] Vol. 19, No. 9-10, S. 78. 
observed psychological and physical aspects, [...] this nature must be something other than >psychological or >physical $<$ as normally understood. ${ }^{78}$

So ist bspw. nach Whiteheads Verständnis das Mentale, in seiner grundlegendsten, rudimentärsten Form, eine intrinsisch-temporäre Erscheinung eines >physikalischen $<$ Ereignisses ${ }^{79}$ oder nach Weekes ist ein >physikalisches Ereignis, in diesem erweiterten Sinne, zugleich ein >mentales< Ereignis, dem einfach ein komplexes Umfeld fehlt. ${ }^{80}$ Alle fundamentalen Einheiten der Natur haben dieser Auffassung zufolge (proto-) phänomenale Eigenschaften, aber nur in komplexen Umgebungen sind sie »hinreichend reichhaltig« (»rich enough « $)^{81}$ um offensichtlich als >bewusstes Erleben< gelten zu können. ${ }^{82}$ Ein >mentales< Ereignis, das als >bewusstes Erleben « gelten kann, ist nach Weekes also gleichzeitig ein >physikalisches< Ereignis, das ein komplexes Umfeld hat. Nach einer solchen Konzeption unterscheidet sich Physikalisches und Mentales also nicht wesentlich: »[...] the mental is, to all appearances, the same as what we normally mean by the physical $\ll$ «. ${ }^{83}$ Mit dergestaltigen Geist- bzw. MaterieKonzeptionen, hat man nicht mehr - wie mit der Prämisse, dass physikalische Phänomene irgendwie >grundlegender< sein müssten als mentale Phänomene (bzw. vice versa) - einen Zwang zum Reduktionismus und deshalb auch nicht mehr die Problematik der Frage, wie die eine Eigenschaft auf die andere reduzierbar sein könnte. Gerade deshalb könnten neutralmonistische und panpsychistische Konzeptionen eine Grundlage für eine Theorie des Geistes darstellen, die sowohl dem Desiderat physikalischer als auch phänomenaler Adäquatheit standhält.

So können wir, sogar mit Pauen konstatieren, dass der Anschein einer grundsätzlichen Verschiedenheit von Qualia und physikalischen Eigenschaften auf

78 Velmans, Max: »Reflexive Monism. Psychophysical Relations among Mind, Matter, and Consciousness «, Journal of Consciousness Studies, [2012] Vol. 19, No. 9-10, S. 154 [Hervorhebung im Original].

79 Vgl. Whitehead, Alfred N.: Process and reality: An essay in cosmology, Cambridge, Massachusetts: The University press 1929, S. 290-293. [Zitiert nach Weekes, Anderson: »The Mind-Body Problem and Whitehead's Non-reductive Monism«, Journal of Consciousness Studies, [2012] Vol. 19, No. 9-10, S. 63.]

8 o Vgl. ebd.

81 Ebd.

82 So konstatiert Weekes: »Furthermore, it follows from this interpretation of mental composition that all fundamental units of nature do indeed have experience, but that only in the context of complexly-structured environments can it be rich enough to distinguish itself and become apparent «. Ebd.

83 A.a.O., S. 62 [Hervorhebung im Original]. 
einer kognitiven Illusion beruht. ${ }^{84}$ Pauen sieht die Ursache dafür jedoch in der Art und Weise, wie wir Qualia gewöhnlich begreifen und meint, dass dieser Begriff im Rahmen des Materialismus rekonstruiert und Qualia in der Folge deshalb erklärt werden könnten. ${ }^{85}$ Die von Pauen postulierte Illusion könnte aber genauso im Hinblick auf unsere Vorstellungen über das, was wir gewöhnlich >Physikalisches $<$ bzw. >Materielles $<$ nennen, bestehen. Dann aber wäre es hilfreich, wenn wir nicht nur unseren Qualia-Begriff, sondern auch unseren Materie-Begriff revidieren würden. Dies führt uns zum nächsten Abschnitt, in welchem ich auf die mit diesen Ansätzen verbundenen Materie-Konzeptionen kurz eingehen werde; in Abschnitt 10.4 erörtere ich dann einige durch sie implizierte Bewusstseins-Konzeptionen.

\subsubsection{Revisionistische Ansätze zum Materie-Begriff}

Das oben erwähnte, vielleicht nur scheinbare Dilemma, entweder das Desiderat phänomenaler Adäquatheit zu ignorieren oder sich die Probleme eines ontologischen Dualismus aufuladen, scheint im Wesentlichen die Schwierigkeit des hard problem auszuzeichnen. Dieser Schwierigkeit könnte jedoch eine metaphysische Fehlannahme zugrunde liegen, die darin besteht, dass Mentales und Physikalisches als zwei ontologische Kategorien definiert werden, die sich gegenseitig ausschliessen - als lebten wir in zwei Welten. ${ }^{86}$

Die Konzeption einer vollständig >objektiven<, physikalischen Realität kann aber nur auf der Grundlage einer theoretisch zwar konstruierbaren, aber epistemisch unerreichbaren, ${ }^{87}$ vollständigen Exklusion von subjektiven Phänomenen aus der Welt zustande kommen. Sowohl die Konzeption einer bewusstseinsunabhängigen, gänzlich >objektiven<, physikalischen Realität als auch die damit verbundene Exklusion von subjektiven Phänomenen, sind paradoxerweise mentale Konstrukte. Nichtsdestotrotz wird mit diesen Konstruktionen versucht, Phänomene des Erlebens und des Bewusstseins in

84 Vgl. dazu Pauen, Michael / Schütte, Michael / Staudacher, Alexander [Hrsg.]: Begriff, Erklärung, Bewusstsein. Neue Beiträge zum Qualia-Problem, Paderborn: Mentis 2007, S. 10.

85 Vgl. dazu ebd.

86 Vgl. dazu Searle, der diese Konzeption von zwei sich gegenseitig ausschliessenden Kategorien von Entitäten in enger Verbindung zum Problem der kausalen Beziehung zwischen Geistigem und Physischen bzw. zum hard problem sieht. Searle, John R. / [Übers.: Gavagai, Harvey]: Intentionalität. Eine Abhandlung zur Philosophie des Geistes, Frankfurt am Main: Suhrkamp [zuerst 1989, engl.]/1991, S. 336 .

87 Vgl. dazu auch Roth, Gerhard: Das Gehirn und seine Wirklichkeit. Kognitive Neurobiologie und ihre philosophischen Konsequenzen, Frankfurt am Main: Suhrkamp 1994, S. 332-333. 
den Bereich des Mentalen abzuschieben - gerade daraus entsteht, Nagel zufolge, das hard problem. 88

Diese metaphysische Fehlannahme von gänzlich >objektivenく Phänomenen, die irgendwie >wirklicher sein sollen, als >absolut subjektive< Phänomene müsste also dahingehend korrigiert werden, dass Physikalisches nicht mehr durch die erwähnten Konstrukte künstlich von mentalen Phänomenen getrennt wird bzw. Bewusstsein nicht mehr in einfach den Bereich des Mentalen abgeschoben wird. Eine Möglichkeit, die aus dieser künstlichen Trennung folgenden Probleme zu vermeiden, besteht also darin, Bewusstsein in unserer Konzeption über das, was wir >Physikalisches $<$ nennen, einzubeziehen. ${ }^{89}$ Weil das vom materialistischen Monismus nie geleistet wurde, können wir mit Nagel, Strawson und McGinn konstatieren, dass gerade auch die Inadäquatheit solch herkömmlicher Konzeptionen über Physikalisches das hard problem erzeugen könnte..$^{90}$ Mit Strawson lässt sich denn auch das Versagen, materialistischmonistischer Konzeptionen des Geistes, das Explanandum des hard problem mit einem nicht-phänomenalen Explanans zu verbinden, damit erklären, dass unser Verständnis über das Physikalische sogar radikal falsch sein könnte:

[...] we must be radically ignorant of the nature of the physical if materialism is true. The main ground for this agnostic materialism is the belief that the apparent difficulty of the mind-body problem cannot plausibly be thought to stem from any hopeless inadequacy or error in our concepts of the mental (as Dennett, for example, supposes) [...]. [...] the failure of these concepts to connect satisfactorily with our concepts of the non-experiential $[\ldots]$ must therefore stem from some other sort of radical [...] inadequacy in our concepts of the nature of the physical. ${ }^{91}$

88 Vgl. dazu Nagel, Thomas: »Consciousness and Objective Reality«, in: The mind-body problem. A guide to the current debate, Warner, Richard / Szubka, Tadeusz [Hrsg.], Cambridge, Massachusetts: Blackwell 1994, S. 66.

89 Vgl. dazu z.B. Nagel, Thomas: »Conceiving the Impossible and the Mind-Body Problem«, Philosophy, [1998] Vol. 73, No. 285, S. 341, 342 und 346.

9o Vgl. dazu Strawson, Galen: »The Experiential and the Non-experiential«, in: The mindbody problem. A guide to the current debate, Warner, Richard / Szubka, Tadeusz [Hrsg.], Cambridge, Massachusetts: Blackwell 1994, S. 7o. Vgl. auch McGinn, Colin: Basic structures of reality. Essays in meta-physics, New York: Oxford University Press 2011, S. 20. Vgl. ebenfalls Nagel, Thomas: »Consciousness and Objective Reality«, in: The mind-body problem. A guide to the current debate, Warner, Richard / Szubka, Tadeusz [Hrsg.], Cambridge, Massachusetts: Blackwell 1994, S. 66.

91 Strawson, Galen:»The Experiential and the Non-experiential«, in: The mind-body problem. A guide to the current debate, Warner, Richard / Szubka, Tadeusz [Hrsg.], Cambridge, Massachusetts: Blackwell 1994, S. 70. 
So sind auch Robinson zufolge die mysteriös erscheinenden Eigenschaften des Mentalen vielleicht nur die Folge unserer »Alltagsintuitionen« (»commonsense view « $)^{92}$ über das Physikalische. ${ }^{93}$ Insofern könnte eine Lösung zum hard problem - neben der Notwendigkeit eines phänomenal adäquaten QualiaBegriffs - zusätzlich darin bestehen, alte Grundintuitionen ${ }^{94}$ bezüglich dessen, was wir >Physikalisches< bzw. >Natur< nennen, zu überholen: »[...] the point being made is simply that in discussing the relation between phenomenology and naturalism we shouldn't make the mistake of letting the concept of nature remain unexamined «.95

Demgegenüber gehen reduktive Theorien des Geistes, offenbar von einer epistemisch zugänglichen, aber paradoxerweise gleichzeitig bewusstseinsunabhängigen physikalischen Realität aus, die als isolierte ontologische Kategorie vorgestellt wird. Aufgrund einer solchen Konzeption sind sie gezwungen, subjektive Phänomene auszuschliessen und müssen daher auch, die aus phänomenaler Inadäquatheit resultierende Last der Irrelevanz ihrer Explanantia tragen.

Im Gegensatz zu solchen physikalistisch-monistischen Reduktionismen kann man mit Strawson jedoch konstatieren, dass nichts, was wir über das Physikalische wissen, gegen die Existenz des Phänomenalen spricht. ${ }^{96}$ So ist nach dem reflexiven Monismus weder die Dritte-Person- auf die Erste-PersonPerspektive noch die Erste-Person- auf die Dritte-Person-Perspektive reduzierbar, sondern sie gelten als wechselseitig irreduzibel (»mutually irreducible «). ${ }^{97}$ Deshalb ist diese Form des neutralen Monismus auch nicht gegen jegliche Spielart des Physikalismus, sondern nur gegen reduktive Formen des Physikalismus, welche die Erste-Person-Perspektive ignorieren oder leugnen:

[...] RM [reflexiver Monismus] does not accept that a third-person, external observer's perspective is the only useful perspective from which to view consciousness, mind, and the surrounding world, and hence rejects reductive physicalism

92 Robinson, Howard: »Dualism«, in: The Stanford Encyclopedia of Philosophy, 2013, Online: http://plato.stanford.edu/archives/fall2o13/entries/dualism/ [zuletzt geprüft am 25.09.2018], Abschnitt 5.1.

93 Vgl. ebd.

94 Vgl. dazu auch Feser, Edward: »Qualia. Irreducibly Subjective but not Intrinsic«, Journal of Consciousness Studies, [2001] Vol. 8, No. 8, S. 6, 9 und 13.

95 Zahavi, Dan: »Naturalized Phenomenology«, 2009, Online: http://cfs.ku.dk/staff/zahavipublications/naturalized_phenomenology.pdf [zuletzt geprüft am 25.09.2018], S. 8.

96 Vgl. dazu Strawson, Galen: »Realistic Monism. Why Physicalism Entails Panpsychism«, Journal of Consciousness Studies, [2006] Vol. 13, No. 10-11, S. 4.

97 Velmans, Max: »Reflexive Monism. Psychophysical Relations among Mind, Matter, and Consciousness «, Journal of Consciousness Studies, [2012] Vol. 19, No. 9-10, S. 162. 
and functionalism. What it is like to experience the universe from a first-person perspective is also an aspect of the universe $[\ldots] .^{98}$

Eine solche, nicht-reduktive Sichtweise auf das hard problem, ist deshalb weder >anti-physikalistischく noch substanzdualistisch. Epistemologisch betrachtet, ist für sie zwar eine Form des Perspektiven-Dualismus zulässig, aber kein ontologischer Dualismus, der Mentales und Physikalisches als sich ausschliessend konzeptualisiert; und auch kein Monismus, welcher versucht, Mentales auf Physikalisches zu reduzieren bzw. Physikalisches von mentalen Eigenschaften $\mathrm{zu}$ isolieren.

\begin{abstract}
Viewed from an external third-person perspective the subject's mental states appear to be physical states in her brain; viewed from the subjects' first-person perspective these states appear in the form of experiences [...]. According to reflexive monism this simply means that a given subject's mental states can be known in two different ways - a form of ontological monism combined with epistemological dualism. But note that neither observer-observed dualism, nor epistemological dualism entails substance dualism. ${ }^{99}$
\end{abstract}

Derartige Spielarten des Physikalismus, die nicht am Desiderat phänomenaler Adäquatheit scheitern, grenzt Chalmers, mit dem Begriff »Naturalismus «, ${ }^{100}$ vom (reduktionistischen) »Materialismus «101 im üblichen Sinne ab. Folglich scheitern nur reduktionistische Formen des Physikalismus am Desiderat phänomenaler Adäquatheit: »Sometimes >naturalism< is taken to be synonymous with >materialism<, but it seems to me that a commitment to a naturalistic understanding of the world can survive the failure of materialism $\ll^{102}$

Sonach lässt sich auch einwenden, dass die Behauptung, Phänomenales lasse sich (im Sinne logisch zwingender Supervenienz) auf >Physikalisches bzw. >Materielles< reduzieren oder sei gar mit ihm identisch $(\Psi=\Phi)$, mit Vorsicht zu geniessen ist, da es nämlich gar nicht so klar ist, was mit diesen Begrifflichkeiten genau gemeint ist. Deshalb konstatiert McGinn, dass die Position des >Physikalismus ziemlich schlecht definiert wird: »[...] the alleged doctrine of >physicalism $<$ is ill defined because there is no workable notion of

\footnotetext{
$98 \quad$ Ebd.

99 A.a.O., S. 163 [Hervorhebung im Original].

100 Chalmers, David J.: The conscious mind. In search of a fundamental theory, New York: Oxford University Press 1996, S. 128.

101 Ebd.

102 Ebd.
} 
the >physical to appeal to «. ${ }^{103}$ So ist nach McGinn eine derartige Behauptung entweder einfach »falsch «104 oder sonst ein ziemlich »leeres «105 Statement. Denn es hängt stark davon ab, was wir unter dem, a prima vista so zweifellos klaren Begriff >Physikalisches` verstehen: entweder wir meinen das, was auf der Grundlage von momentanen physikalischen Kenntnissen unter ihm verstanden wird und insoweit nicht kompatibel mit Phänomenalem zu sein scheint oder man verweist damit auf etwas, das in einer zukünftigen, vollkommenen und idealen Physik unter diesem Begriff verstanden werden könnte. ${ }^{106}$ Erstere Variante der Behauptung $>\Psi=\Phi<$ mit einem zugrundeliegenden konservativen Physik-Begriff würde für >Physikalisten « in diesem Sinne eine evasive Haltung phänomenaler Indifferenz erzwingen; die zweite Variante käme aber etwa folgender trivialer Aussage nahe: >Hätten wir eine ideale, zukünftige Wissenschaft, die alles beschreiben könnte, dann könnte sie auch das hard problem erklären, diese ideale Wissenschaft würden wir dann auch >Physik« nennen - also kann man vielleicht einmal bewusstes Erleben physikalistisch erklären ${ }^{107}$

Die Gefechte zwischen so genannten >Physikalisten< und >Anti-Physikalisten< in der Philosophie des Geistes könnten sich sonach als Dispute erweisen, bei denen gar nicht klar ist, wo eigentlich das Schlachtfeld ist: »The philosopher of mind with an interest in physics my also wonder whether >physicalism $<$ or >materialism $<$ is a doctrine to defend. Certainly a doctrine $\underline{s o}$ named has been defended « ${ }^{108}$ Eine Revision, die von zwei Seiten her ansetzt, nämlich die Revision allzu einfacher, nicht hinterfragter Intuitionen über das, was wir $>$ Materielles $<$ resp. $>$ Physikalisches $<$ nennen und eine Revision von viel zu engen, der Eliminierbarkeit dienlichen (bzw. der Explizierbarkeit dienlichen, transformierten) Qualia-Begriffen, könnte daher die Vorarbeit leisten, welche mindestens eine Basis schaffen würde, das hard problem erst einmal in seiner Brisanz zu verstehen. In der Folge böten sich Lösungsmöglichkeiten an, die nicht in ein Dilemma zwischen dem Verlust des Explanandums und einem ontologischen Dualismus führen würden. ${ }^{109}$

103 McGinn, Colin: Basic structures of reality. Essays in meta-physics, New York: Oxford University Press 2011, S. 8. Neben dem Umstand, dass die (vermeintliche) Doktrin des >Physikalismus« schlecht definiert wird, bietet sie McGinn zufolge auch nichts brauchbares, worauf man sich berufen könnte.

104 Ebd.

105 Ebd.

106 Vgl. dazu auch ebd.

107 Kein Zitat, sondern fiktive Rede von mir. Vgl. dazu ebenfalls a.a.O., S. 7-8 und 1 o.

108 A.a.O., S. 8 und 10 [Hervorhebung kursiv im Original; Hervorhebung kursiv und unterstrichen von E. E.].

109 Ebd. 
Im Rahmen dieser Arbeit kann ich jedoch nicht weiter auf Revisionen unserer Physikalismus- bzw. Materie-Konzeptionen, wie z.B. die von Russells neutralem Monismus, eingehen. ${ }^{110}$ An dieser Stelle sei lediglich angemerkt, dass die Beweislastzuschiebung von materialistisch-monistischen Positionen, im Hinblick auf zukünftig plausibel erscheinende Reduktionen von Qualia, auf der Grundlage der hier aufgeführten Argumente, genauso gut umgekehrt werden kann. So stellt bspw. Mandik, ganz im Sinne reduktionsoptimistischer Positionen fest: »Why think that theoretical advances might not alter our conception of qualia in similar ways? [...] We do not yet possess the requisite brain theory; therefore, we do not yet know if the physical entails the phenomenal «.111 Wenn wir die Beweislast jedoch umkehren, könnten wir genauso folgende Frage stellen: >Why think that theoretical advances might not alter our conception of matter? We do not yet posses the requisite matter theory; therefore, we do not yet know if the phenomenal is as basic as the physical..112

\subsection{Emergenzproblem versus Bewusstsein als fundamentale Eigenschaft}

Die verschiedenen Zugänge zum hard problem lassen sich ebenfalls in solche mit und solche ohne Rückgriff auf eine Emergenzkonzeption, ${ }^{113}$ im Hinblick auf das Phänomen >Bewusstsein< aufteilen. Wenn eine Theorie des Geistes

110 Vgl. dazu Russell, Bertrand: »Excerpt from >Mind and matter«, in: Philosophy of mind. Critical concepts in philosophy. Volume 1: Foundations, Crawford, Sean [Hrsg.], London: Routledge \& Kegan Paul [zuerst 1956]/2011, S. 167-174.

111 Mandik, Pete / Weisberg, Josh: »Type-q materialism«, 2008, Online: http://www. petemandik.com/philosophy/papers/typeq.pdf [zuletzt geprüft am 13.09.2018], S. 8 [Hervorhebung E. E.].

112 Kein Zitat, sondern meine beweislastbezogene Umkehrung des Zitates von Mandik, vgl. dazu a.a.O., S. 8.

113 Emergenztheorien gehen, vereinfacht gesagt davon aus, dass das Ganze mehr ist als die Summe seiner Teile und dass aus dem Zusammenwirken von Teilen eines komplexen Systems Phänomene entstehen können, die nicht vorhersehbar oder sogar ontologisch neuartig sind. Vgl. dazu Mill, John S.: A system of logic ratiocinative and inductive. Being a connected view of the principles of evidence and the methods of scientific investigation, London: Longmans, Green \& Co [zuerst 1843]/19o6. Vgl. ebenfalls Broad, Charles D.: The mind and its place in nature, London: Routledge \& Kegan Paul 1925. Zur Konzeption der Emergenz von Lewes, Broad und Mill vgl. auch O'Connor, Timothy / Wong, Hong Yu: »Emergent Properties«, in: The Stanford Encyclopedia of Philosophy, 2012, Online: http:// plato.stanford.edu/archives/spr2o12/entries/properties-emergent/ [zuletzt geprüft am 28.o9.2018], Abschnitt Einleitung, 1.1 und 1.4. 
nicht, wie bspw. der Panpsychismus, davon ausgeht, dass Bewusstsein eine fundamentale Eigenschaft des Universums ist, dann muss sie entweder Bewusstsein leugnen ${ }^{114}$ oder sie muss sich (irgendwo auf dem Kontinuum zwischen phänomenalen und nicht-phänomenalen Eigenschaften) auf die Konzeption der Emergenz berufen.

$[\ldots]$ if one does not place mind at the very foundation, and in fact regards mentality to be a feature of systems of non-mentalistic entities, then one is an emergentist. Crudely put, someone who believes that amoebas have experiences, but that quarks and electrons, which ultimately constitute amoebas, do not is no panpsychist.115

So müssen der Epiphänomenalismus, die nicht-reduktiven Varianten des Funktionalismus und die des schwachen Repräsentationalismus auf die eine oder andere Form von Emergenz zurückgreifen. Im Zusammenhang mit dem nicht-reduktiven Funktionalismus konstatiert z.B. Chalmers: $»[\ldots]$ consciousness arises from functional organization but is not a functional state «.116 Für den schwachen Repräsentationalismus, wie dem von Block, Chalmers, Loar und Peacocke sind Qualia nicht zwingend identisch mit einer Repräsentation - das bedeutet aber, dass bewusstes Erleben, als ontologisch >neue< Eigenschaft, ${ }^{117}$ bei einer bestimmten Form von repräsentationalem Gehalt irgendwie entstehen bzw. emergieren muss. ${ }^{118}$

114 Bzw. es mit >rein< Physikalischem identifizieren, vgl weiter unten.

115 Seager, William / Allen-Hermanson, Sean: »Panpsychism«, in: The Stanford Encyclopedia of Philosophy, 2013, Online: http://plato.stanford.edu/archives/fall2013/entries/panpsychism/ [zuletzt geprüft am 25.09.2018], Abschnitt 1.o.

116 Chalmers, David J.: The conscious mind. In search of a fundamental theory, New York: Oxford University Press 1996, S. 249. Zum nicht-reduktiven Funktionalismus vgl. auch Block, Ned J.: »Inverted Earth«, in: Philosophy of mind. Critical concepts in philosophy. Volume 4: Consciousness, Crawford, Sean [Hrsg.], London: Routledge \& Kegan Paul [zuerst 1990]/2011, S. 121.

117 Vgl. dazu auch Lycan, William: »Representational Theories of Consciousness «, in: The Stanford Encyclopedia of Philosophy, 2008, Online: http://plato.stanford.edu/archives/ fall2008/entries/consciousness-representational/ [zuletzt geprüft am 28.09.2018], Abschnitt 2.1.

118 Vgl. dazu Chalmers, David J.: The conscious mind. In search of a fundamental theory, New York: Oxford University Press 1996, S. 377. Oder: Chalmers, David J.: »The Representational Character of Experience«, in: The future for philosophy, Leiter, Brian [Hrsg.], Oxford: Clarendon Press 2004. Oder: Block, Ned J.: »Mental Paint and Mental Latex«, Philosophical Issues, [1996] Vol. 7: Perception, S. 19-49. Vgl. ebenfalls Pitt, David: »Mental Representation«, in: The Stanford Encyclopedia of Philosophy, 2008, Online: http://plato.stanford.edu/archives/fall20o8/entries/mental-representation [zuletzt geprüft am 25.09.2018], Abschnitt 4.0. 
Der Epiphänomenalismus leugnet zwar ebenfalls nicht das Explanandum bewussten Erlebens. ${ }^{119}$ Er definiert jedoch mentale Zustände als kausal irrelevante Eigenschaften, ${ }^{120}$ die vollständig durch physikalische bzw. neurophysiologische Ereignisse verursacht werden können. ${ }^{121}$ Da nach dem Epiphänomenalismus Physikalisches und Mentales dennoch zu unterscheiden sind, ${ }^{122}$ muss auch er auf irgendeine Form von Emergenz zurückgreifen, die >erklärt<, warum bestimmte physikalische Ereignisse mentale Phänomene hervorrufen können.

Doch der Rückgriff auf Emergenz, im Hinblick auf Bewusstsein, ist nicht ohne Probleme: wo lässt sich diese Grenze ziehen, zwischen phänomenalen und nicht-phänomenalen Eigenschafen - und mit welcher Begründung? Warum und ab welchem Grad von Komplexität soll in einem Organismus aus vollständig >nicht-phänomenalem Material« Bewusstsein entstehen? Nach Strawson ist Emergenz sogar eine »magische ${ }^{123}$ Konzeption, die das Verhältnis

119 Der Epiphänomenalismus wurde im späten 18. und späten 19. Jahrhundert entwickelt als Konsequenz von zwei scheinbar unvereinbaren Ansichten über die Welt: 1.) Eine wachsende Zuversicht, dass die Welt eine reine Welt von Kausalitäten ist und dass die Welt kausal geschlossen ist. 2.) Die Überzeugung, dass Physikalisches und Mentales zu unterscheiden sind. Mit dem Epiphenomenalismus wollte man diese zwei (scheinbar) konfligierenden Überzeugungen insofern vereinen, dass nach ihm Mentales keine kausale Wirkung auf Physikalisches hat, was trotz Nicht-Identität von Mentalem und Physikalischem die kausalen Geschlossenheit der Welt nicht verletztn würde. Somit nähert sich der Epiphänomenalismus dem hard problem vor allem durch die Problemoptik der kausalen und weniger durch die der phänomenalen Achse. Vgl. dazu auch Huxley, Thomas H.: »On the hypothesis that animals are automata, and its history«, in: Collected Essays, Volume I, Method and Results, Huxley, Thomas H. [Hrsg.], London: Macmillan [zuerst 1874]/1893, S. 240-243. Vgl. ebenfalls Walter, Sven: »Epiphenomenalism«, in: The Oxford Handbook of Philosophy of Mind, McLaughlin, Brian P. / Beckermann, Ansgar / Walter, Sven [Hrsg.], Oxford: Oxford University Press 2009, S. 85-94.

120 Genauer: kausal irrelevant für Physikalisches bzw. für unser Verhalten. Nach dieser Konzeption scheint es also nur so, dass unser Verhalten von einer Überzeugung oder Empfindung verursacht wurde. Vgl. dazu auch Schröder, Jürgen: »Qualia und Physikalismus «, Journal for General Philosophy of Science, [1997] Vol. 28, No. 1, S. 166-177.

121 Diese Prämisse lässt nach Walter allerdings die Möglichkeit für mentale Ereignisse offen, die nicht durch physikalische Ereignisse ausgelöst werden, solange diese mentalen Ereignisse keine kausalen Auswirkungen auf Physisches haben. Mentale Ereignisse könnten demnach zwar andere mentale Ereignisse verursachen, im Hinblick auf Physisches bleiben sie jedoch wirkungslos. Vgl. dazu Walter, Sven: »Epiphenomenalism«, in: The Oxford Handbook of Philosophy of Mind, McLaughlin, Brian P. / Beckermann, Ansgar / Walter, Sven [Hrsg.], Oxford: Oxford University Press 2009, S. 86.

122 Vgl. ebd.

123 Strawson, Galen: »Realistic Monism. Why Physicalism Entails Panpsychism«, Journal of Consciousness Studies, [2006] Vol. 13, No. 10-11, S. 20. 
zwischen Phänomenalem und Nicht-Phänomenalem zu einem »garantierten Mysterium $\ll^{124}$ mache:

Some who want to talk of experiential properties as >emergent $<$ relative to nonexperiential properties may feel that this represents their position accurately, although it seems to turn the relation between the experiential and the nonexperiential into a guaranteed mystery, where before it was merely a mystery. ${ }^{125}$

Die Konzeption der Emergenz leistet in explanatorischer Hinsicht tatsächlich wenig und füllt wohl eher entweder eine epistemische (schwacher Emergentismus) oder eine ontologische Lücke (starker Emergentismus).126 Warum soll über einer komplizierten Anordnung von Atomen bzw. Makromolekülen, wie z.B. bei einer Amöbe, plötzlich Bewusstsein emergieren und bei einer anderen, einfacheren Anordnung nicht? Wenn emergente Eigenschaften ohne definitiv zu eruierende Gründe entstehen können, müssten wir nach Strawson sogar einräumen, dass unter Umständen aus Blei vielleicht doch einmal Gold emergieren könnte:

If emergence can be brute, then it is fully intelligible to suppose that nonphysical soul-stuff can arise out of physical stuff - in which case we can't rule out the possibility of Cartesian egos even if we are physicalists. [...] We will certainly have to view with equanimity all violations of existing laws of (non-experiential) physics, dross turning adventitiously into gold $[\ldots]]^{127}$

124 Strawson, Galen:»The Experiential and the Non-experiential«, in: The mind-body problem. A guide to the current debate, Warner, Richard / Szubka, Tadeusz [Hrsg.], Cambridge, Massachusetts: Blackwell 1994, S. 77.

125 Ebd.

126 Bei Emergenztheorien lassen sich schwache (epistemische) und starke (ontologische) Formen unterscheiden. Ein starker Emergentismus geht davon aus, dass aufgrund der Emergenz vollständig neue Eigenschaften (oder sogar emergente Substanzen) entstehen, während man bei schwachen Formen von Emergenztheorien nur davon ausgeht, dass die emergenten Eigenschaften aus einem präemergenten Zustand nicht voraussagbar sind. Zu starken Emergentismen vgl. O'Connor, Timothy / Wong, Hong Yu: »Emergent Properties«, in: The Stanford Encyclopedia of Philosophy, 2012, Online: http:// plato.stanford.edu/archives/spr2012/entries/properties-emergent/ [zuletzt geprüft am 28.09.2018], Abschnitt 3.1 und 5.0. Zu schwachen, epistemologischen Emergenztheorien vgl. Alexander, Samuel: Space, time and deity. The Gifford lectures at Glasgow 1916-1918, London: Macmillan [zuerst 1920]/1934. Vgl. auch O'Connor, Timothy / Wong, Hong Yu: »Emergent Properties«, in: The Stanford Encyclopedia of Philosophy, 2012, Online: http:// plato.stanford.edu/archives/spr2o12/entries/properties-emergent/ [zuletzt geprüft am 28.09.2018], Abschnitt 2.0.

127 Strawson, Galen: »Realistic Monism. Why Physicalism Entails Panpsychism«, Journal of Consciousness Studies, [2006] Vol. 13, No. 10-11, S. 19 [Hervorhebung im Original]. 
Um das Emergenzproblem von phänomenalen Eigenschaften zu umgehen, kann man erstens auf reduktionistische Monismen ausweichen, d.h. entweder nicht-phänomenale Eigenschafen auf phänomenale Eigenschafen reduzieren oder vice versa. Erstere Form des Reduktionismus geht davon aus, dass nur Bewusstsein und Qualia existieren. Folgen wir diesem Reduktionismus, können wir analog zum Eliminativismus behaupten, dass uns die Alltagsintuition verleitet, sinnlich-phänomenale Erlebnisse so zu interpretieren, als ob es tatsächlich irgendwo >da Draussen< materielle Objekte gäbe - der Eindruck, dass wir intersubjektiv Erleben und dass es so etwas wie >Materie< unabhängig von Bewusstsein gibt, wäre diesem Reduktionismus zufolge einfach eine Illusion.

Was für diese Form des Reduktionismus sogar etwas mehr spricht als für sein materialistisch-eliminativistisches Pendant, ist das Argument, dass wir in erster Instanz nur um unser Erleben wissen. So lässt es sich nach Feser, aufgrund der Korrelationen von funktionalen Zuständen und Qualia auch gut begründen, dass wir unsere Konzeption von Materie (oder von funktionalen Zuständen) auf der Grundlage von Qualia formen. ${ }^{128}$ Nach dieser Konzeption bzw. >umgekehrten< Reduktion ist der qualitative Charakter des Erlebens grundlegend für kausale und funktionale Rollen von Hirnzuständen bzw. nicht trennbar von letzteren.

$[\ldots]$ it is generally not realized that when it comes to identifying qualia and features of brain states, or qualia and features of functional states, the sreduction< can go two ways and that given the indirect realist claim that all we know directly are qualia in the first place, the Russellian way - or better, the (revised) functionalist way I have suggested - is the more plausible reduction. ${ }^{129}$

Eine Lösung des Emergenzproblems, welche Nicht-Phänomenales auf Phänomenales reduziert, ist jedoch, wenn sie strikt monistisch gedacht wird, aber nur zum Preis der Probleme des Idealismus oder gar des Solipsismus zu haben. Dennoch kann man konstatieren, dass dieser Reduktionismus im Hinblick auf das hard problem noch plausibler ist als die Identitätstheorie oder der Eliminativismus, ${ }^{130}$ weil er dem Grundsatz der »Rettung der Phänomene «131 insoweit nachkommt, als er das Explanandum >Bewusstsein< wenigstens nicht

128 Wörtlich: »[...] we have every reason to model our conception of functional states on qualia (just as some Russellians suggest modelling our conception of matter on qualia $[\ldots] \ll$. Feser, Edward: »Qualia. Irreducibly Subjective but not Intrinsic«, Journal of Consciousness Studies, [2001] Vol. 8, No. 8, S. 13 [Hervorhebung im Original].

129 Ebd. [Hervorhebung von E. E.].

130 Vgl. dazu auch ebd.

131 Schildknecht, Christiane: »Der Dualismus und die Rettung der Phänomene«, in: Homo Sapiens und Homo Faber. Epistemische und technische Rationalität in Antike und Gegenwart. 
ignoriert. Auch mit dem Eliminativismus (weil es für diesen gar kein Bewusstsein >gibt<) oder mit der Identitätstheorie (weil Physikalisches als >identisch mit Bewusstsein gilt) lässt sich zwar das Emergenzproblem umgehen. Diese und andere Formen der phänomenalen Indifferenz bzw. des phänomenalen Antirealismus erweisen sich aber, aufgrund der oben besprochenen Probleme und der Irrelevanz ihrer Explanantia bzw. ihrer Beschäftigung mit Explananda, die wenig bis nichts mit dem hard problem zu tun haben, leider auch als ziemlich irrelevant für das hard problem. Beim Eliminativismus sind die Probleme, wie in den Abschnitten 7.1 und 10.2 dargelegt, noch gravierender. Dies ist auch deshalb der Fall, weil er, entgegen dem Desiderat phänomenal Adäquatheit und der damit verbundenen ockhamschen Regel, aufgrund einer Vorentscheidung für eine strikte Form des Monismus, an der Ontologie der exklusiven Existenz von nicht-phänomenalen Entitäten festhält und sich dabei nicht zuletzt auf Hoffnungen beruft, wie z.B.: a.) vielleicht >täuscht< uns unser Erleben nur vor, dass wir erleben; b.) vielleicht gibt es irgendwann einmal zukünftige Erkenntnisse welche die Konzeption der >Nicht-Existenz` unseres Erlebens plausibel machen könnten. ${ }^{132}$

Zweitens hat auch ein ontologischer Dualismus kein Emergenzproblem, weil Bewusstsein als etwas ontologisch Unabhängiges bereits vorausgesetzt wird. Er hat jedoch ein Kausalitäts- bzw. Interaktionsproblem, das darin besteht, wie denn Mentales, wenn es doch etwas vollständig anderes ist als Physikalisches, auf letzteres einwirken kann bzw. vice versa. ${ }^{133}$

Festschrift für Jürgen Mittelstrass, Wolters, Gereon / Mittelstrass, Jürgen [Hrsg.], Berlin: W. de Gruyter 2005, S. 225 .

132 Vgl. dazu auch Abschnitt 4.2.5

133 Vgl. dazu White, Stephen L.: »Curse of Qualia«, Synthese, [1986] Vol. 68, No. 2, S. 355. Vgl. ebenfalls Elitzur, Avshalom C.: »Consciousness makes a difference: A reluctant dualist's confession«, 2009, Online: http://philpapers.org/archive/ELICMA.pdf [zuletzt geprüft am 13.09.2018], S. 11. Vgl. ebenfalls Robinson, Howard: »Dualism«, in: The Stanford Encyclopedia of Philosophy, 2013, Online: http://plato.stanford.edu/archives/fall2013/ entries/dualism/ [zuletzt geprüft am 25.09.2018], Abschnitt 1.1. Zum zeitgenössischen Substanzdualismus von Swinburne vgl. z.B. Swinburne, Richard: »Body and Soul«, in: The mind-body problem. A guide to the current debate, Warner, Richard / Szubka, Tadeusz [Hrsg.], Cambridge, Massachusetts: Blackwell 1994, S. 311-316. 
Die dritte Möglichkeit, das Emergenzproblem zu umgehen, besteht in bestimmten Versionen des neutralen Monismus ${ }^{134}$ und des Panpsychismus, ${ }^{135}$ die Bewusstsein im Sinne einer fundamentalen und sonach nicht-emergenten Eigenschaft des Universums voraussetzen. ${ }^{136}$ Nach Chalmers sollte eine nichtreduktive Theorie des Geistes es denn auch aufgeben, Bewusstsein mit einer noch fundamentaleren Eigenschaft erklären zu wollen. ${ }^{137}$ Bewusstsein ist nach dieser innovativen Betrachtungsweise, analog zu Naturkonstanten wie bspw. der Gravitation, dem Elektromagnetismus, Raum, Zeit oder Materie, nicht auf etwas Fundamentaleres zurückführbar. ${ }^{138}$

Either experiential properties, being natural physical properties, are reducible to other natural physical properties [...] or they are not. But if they are not so reducible, then at least some of them must themselves be fundamental physical properties like (e.g.) extension or electric charge [...]..139

Auch elektromagnetische Phänomene konnten im 19. Jahrhundert nicht auf die bis dato bekannten Kräfte der klassischen Physik reduziert werden, sondern es stellte sich heraus, dass Elektromagnetismus als eine fundamentale Kraft, entgegen dem Prinzip ontologischer Sparsamkeit anerkannt werden musste. ${ }^{140}$

134 Vgl. dazu z.B. Feigl, Herbert: The >Mentak and the >Physicak. The Essay and a Postscript, Minneapolis, Minnesota: University of Minnesota Press [zuerst 1958]/1966. Vgl. auch Russell, Bertrand: The analysis of matter, London: Kegan Paul, Trench, Truber \& Co 1927, S. 389. Vgl. ebenfalls Maxwell, Grover: »Rigid designators and mind-brain identity«, in: Perception and cognition. Issues in the foundations of psychology, Savage, Wade C. [Hrsg.], Minneapolis: University of Minnesota Press 1978.

135 Vgl. z.B. Seager, William / Allen-Hermanson, Sean: »Panpsychism«, in: The Stanford Encyclopedia of Philosophy, 2013, Online: http://plato.stanford.edu/archives/fall2013/ entries/panpsychism/ [zuletzt geprüft am 25.09.2018], Abschnitt 1.o-3.o. Oder: Strawson, Galen: $\gg$ The Experiential and the Non-experiential«, in: The mind-body problem. A guide to the current debate, Warner, Richard / Szubka, Tadeusz [Hrsg.], Cambridge, Massachusetts: Blackwell 1994, S. 69-86. Oder: Strawson, Galen: »Realistic Monism. Why Physicalism Entails Panpsychism «, Journal of Consciousness Studies, [2006] Vol. 13, No. 10-11, S. 3-31.

136 Vgl. dazu auch Seager, William: »Emergentist Panpsychism«, Journal of Consciousness Studies, [2012] Vol. 19, No. 9-10, S. 34. Davon ausgenommen sind jedoch besonders schwache bzw. emergentistische Formen des Protopanpsychismus. Vgl. dazu die Fussnote weiter unten. (Zum emergentistischen Panpsychismus vgl. a.a.O., S. 19, 35 und 37.)

137 Vgl. Chalmers, David J.: The conscious mind. In search of a fundamental theory, New York: Oxford University Press 1996, S. 213.

138 Vgl. ebd.

139 Strawson, Galen:»The Experiential and the Non-experiential«, in: The mind-body problem. A guide to the current debate, Warner, Richard / Szubka, Tadeusz [Hrsg.], Cambridge, Massachusetts: Blackwell 1994, S. 73 .

140 Vgl. Chalmers, David J.: The conscious mind. In search of a fundamental theory, New York: Oxford University Press 1996, S. 127. 
Analog zu diesem historischen Beispiel, dass es zuweilen auch notwendig sein kann, eine den Phänomenen gerecht werdende Überwindung Ontologischer $>$ Geizigkeit< bzw. Fixiertheit anzuwenden, besteht eine plausible Option, das Emergenzproblem zu vermeiden darin, Bewusstsein als eine fundamentale bzw. irreduzible Eigenschaft anzuerkennen. ${ }^{141}$

Denn, wenn Phänomenales aus etwas entstehen kann, so muss dieses retwas in irgend einer Weise schon phänomenal oder mindestens protophänomenal ${ }^{142}$ sein, andernfalls sind phänomenale Realisten gezwungen, sich auf jene seltsame Emergenz zu stützen, die irgendwo an einem Punkt zwischen Phänomenalem und Nicht-Phänomenalem wie ein geheimnisvoller Blitz einschlägt und Bewusstsein hervorzaubert.

So if experience like ours (or mouse experience, or sea snail experience) emerges from something that is not experience $[. .$.$] then that something must already be$ experiential in some sense or other. It must already be somehow experiential in its essential and fundamental nature, however primitively or strangely or (to us) incomprehensibly [...] Otherwise we're back at brutality, magic passage across the experiential/non-experiential divide, something that, ex hypothesi, not even God can understand $[\ldots] .143$

Die Konzeption von Bewusstsein bzw. Phänomenalität als fundamentale und irreduzible Eigenschaft setzt aber, entgegen aller Behauptungen, keinen ontologischen Dualismus voraus, ${ }^{144}$ wenn sie im Sinne eines neutralen Monismus, wie z.B. dem von Russell verstanden wird. Die dem Mentalen und Physikalischen zugrunde liegenden Prozesse sind demgemäss zugleich als

\footnotetext{
141 Vgl. dazu auch ebd.

142 Wenn wir Protophänomenalität in einem engen Sinne verstehen bzw. je stärker wir es von Phänomenalität abgrenzen, umso mehr resultiert daraus jedoch wieder ein Emergenzproblem: wie und warum kann aus Protophänomenalem Phänomenales entstehen. Zum subtilen Emergenzproblem bei einem Panpsychismus mit protopanpsychistischen Ansätzen vgl. auch Seager, William: »Emergentist Panpsychism «, Journal of Consciousness Studies, [2012] Vol. 19, No. 9-10, S. 19, 35 und 37. Zum >Kombinationsproblem<, wie aus protophänomenalen Eigenschaften die uns bekannten phänomenalen Eigenschaften entstehen können, vgl. Alter, Torin / Nagasawa, Yujin: »What is Russellian Monism?«, Journal of Consciousness Studies, [2012] Vol. 19, No. 9-10, S. 91. Zum Protopanpsychismus vgl. auch Chalmers, David J.: »Panpsychism and Panprotopsychism«, 2013, Online: http:// consc.net/papers/panpsychism.pdf [zuletzt geprüft am 13.o9.2018], S. 13-15.

143 Strawson, Galen: »Realistic Monism. Why Physicalism Entails Panpsychism«, Journal of Consciousness Studies, [2006] Vol. 13, No. 10-11, S. 24 und 26 [Hervorhebung im Original].

144 Searle führt diese Idee, wie oben dargelegt, auf unser cartesianisches Erbe zurück. Vgl. Searle, John R.: The mystery of consciousness, New York: New York Review Books 1997, S. 195 .
} 
$>$ physikalisch< und als >mental< zu verstehen. ${ }^{145}$ Die »[...] intrinsische Qualität von physikalischen Ereignissen $[. ..] \ll^{146}$ kann ein mentales Ereignis mit seiner Phänomenalität hervorbringen, während deren extrinsisch-relationale Aspekte das Physikalische instantiieren. ${ }^{147}$

Demgegenüber gehen die Varianten des Panpsychismus bzw. des Panprotopsychismus von fundamentalen physikalischen Eigenschaften aus, die (proto-) phänomenale Eigenschaften haben. ${ }^{148}$ Auch in diesen Ansätzen geht man von fundamentalen und insofern nicht-emergenten phänomenalen oder mindestens protophänomenalen Eigenschaften aus, die nicht mehr auf etwas Grundlegenderes reduziert werden können. So ist für Seager Mentales eine »Kernkomponente« ${ }^{149}$ der Realität: »[...] if mentality is regarded as metaphysically fundamental, then the mental will fail to be ontologically dependent on anything - it will stand as one of the core components of reality «. ${ }^{150}$ Daher muss der Panpsychismus, insbesondere dessen neutralmonistische Variante, keineswegs dem Naturalismus - und schon gar nicht einem revidierten Physikalismus ${ }^{151}$ - widersprechen:

The physical is whatever general kind of thing we are considering when we consider things like tables and chairs and experiential phenomena. It includes everything that concretely exists in the universe. If everything that concretely exists is intrinsically experience-involving, well, that is what the physical turns out to be; it is what energy (another name for physical stuff) turns out to be. This view does not stand out as particularly strange against the background of present-day science, and is in no way incompatible with it. ${ }^{152}$

145 Vgl. Russell, Bertrand: »Excerpt from $>$ Mind and matter««, in: Philosophy of mind. Critical concepts in philosophy. Volume 1: Foundations, Crawford, Sean [Hrsg.], London: Routledge \& Kegan Paul [zuerst 1956]/2011, S. 174.

146 (»the intrinsic quality of physical events«), vgl. Ebd. Genauer müsste man hier wohl von 'physikalisch-mentalen Ereignissen sprechen, da diese nach Russell ja nicht nur dem mentalen, sondern auch dem Physikalischen zugrunde liegen.

147 Vgl. ebd. Vgl. dazu auch Chalmers, David J.: The conscious mind. In search of a fundamental theory, New York: Oxford University Press 1996, S.155. Vgl. ebenfalls Alter, Torin / Nagasawa, Yujin: »What is Russellian Monism?«, Journal of Consciousness Studies, [2012] Vol. 19, No. 9-10, S. 73 .

148 Vgl. Chalmers, David J.: »Panpsychism and Panprotopsychism«, 2013, Online: http:// consc.net/papers/panpsychism.pdf [zuletzt geprüft am 13.09.2018], S. 13 .

149 Seager, William: »Emergentist Panpsychism«, Journal of Consciousness Studies, [2012] Vol. 19, No. 9-10, S. 23.

150 Ebd.

151 Vgl. a.a.O., S. 24.

$15^{2}$ Strawson, Galen: »Realistic Monism. Why Physicalism Entails Panpsychism«, Journal of Consciousness Studies, [2006] Vol. 13, No. 10-11, S. 7-8. 
Die Konzeption von irreduziblen Eigenschaften, die das konstituieren, was wir >Erleben< nennen, erweitert aber freilich ein strikt materialistischmonistisches bzw. physikalistisch-reduktionistisches Weltbild, indem man mit ihr bestreitet, dass sich Bewusstsein auf absolut nicht-phänomenale Entitäten reduzieren lässt.

This form of panpsychism [...] denies that what physics reveals exhausts fundamental nature. Instead, for each physically fundamental entity - whatever those may turn out to be - it assigns some mentalistic property, presumably some unutterably primitive and simple form of consciousness. ${ }^{153}$

Was die Erklärung von mentalen Phänomenen, wie Bewusstsein und Erleben sicher nicht einfacher macht, ist der Umstand, dass Bewusstsein selbst den Nabel unseres epistemischen Universums bildet: »[...] conscious experience is at the very center of our epistemic universe $\ll .154$ Wenn mentale Phänomene herausfinden möchten, wie sie selber zustande kommen, dann ist das nach Roth ein »fundamental selbstreferentielles Unterfangen «.155 Diese Problematik bestünde sogar dann, wenn sie `nichts anderes als« blosse Neuronenaktivität oder sogar snicht wirklich existierend` wären: Auch dann unterstünden wir dem epistemischen Zirkel, dass mit Neuronenaktivität identische (`nichtwirklich-existierende<) mentale Phänomene das Wesen der mit Neuronenaktivität identischen (>nicht-wirklich-existierenden`) Phänomene herauszufinden versuchten.

Wir können uns dem Phänomen des Phänomenalen nicht wirklich >objektiv<, das heisst, unter Ausschluss des Mentalen selbst nähern. Insbesondere bei mentalen Phänomenen, gälte es demnach, sich einzugestehen, dass sie nie im Sinne objektiver »[...] überzeugungsfreier wissenschaftlicher Beobachtungsdaten $[\ldots] \ll^{156}$ verstanden werden können. Wenn sich schon bei Phänomenen, die nicht dem oben erwähnten epistemischen Zirkel unterliegen, feststellen lässt, dass unsere auf sie bezogene epistemische Situation nicht zwingend der $»[\ldots]$ neuzeitlichen Annahme einer interpretationsfreien

153 Seager, William: »Emergentist Panpsychism«, Journal of Consciousness Studies, [2012] Vol. 19, No. 9-10, S. 24.

154 Chalmers, David J.: The conscious mind. In search of a fundamental theory, New York: Oxford University Press 1996, S. 74 .

155 Roth, Gerhard: Das Gehirn und seine Wirklichkeit. Kognitive Neurobiologie und ihre philosophischen Konsequenzen, Frankfurt am Main: Suhrkamp 1994, S. 23.

${ }_{15} 6$ Schildknecht, Christiane: »Der Dualismus und die Rettung der Phänomene«, in: Homo Sapiens und Homo Faber. Epistemische und technische Rationalität in Antike und Gegenwart. Festschrift für Jürgen Mittelstrass, Wolters, Gereon / Mittelstrass, Jürgen [Hrsg.], Berlin: W. de Gruyter 2005, S. 230. 
Erfahrungsbasis $[\ldots] \ll^{157}$ entspricht, um wie viel kritischer gälte es dann, im Hinblick auf unseren epistemischen Zugang zu mentalen Phänomenen wie Bewusstsein und Erleben zu sein. Sich der Problematik und kontingenten Verfasstheit unserer epistemischen Situation bewusst zu sein, ist insofern weniger spekulativ, als die Idee einer interpretationsfreien Basis der Erfahrung und der damit verbundene Versuch, die Welt und das Mentale bewusstseinsunabhängig zu beschreiben:

Das Resultat ist eine Beschreibung der Welt, wie sie denjenigen erscheint (und nicht wie sie ohne Bezug auf unsere Erfahrung an und für sich sein könnte), deren epistemische Verfasstheit der unsrigen gleicht. ${ }^{158}$

Selbst wenn sich, entgegen meiner Ausführungen in Abschnitt 10.2, das Patt zwischen Reduktionsoptimisten und Reduktionsskeptikern, trotz der Ubiquität phänomenalen Erlebens nicht verschieben würde, so spräche nicht nur der epistemische Zirkel im Hinblick auf mentale Phänomene, sondern auch unsere epistemische Situation überhaupt, für dementsprechend vorsichtigere und skeptischere Positionen. Vorsichtiger z.B. als solche, die sich beim hard problem, aufgrund jenes Ideals einer bewusstseinsunabhängigen, »objektivistischen ${ }^{159}$ Beschreibung der Welt, auf strikt monistisch-materialistische Ontologien zurückziehen. Aufgrund desselben Ideals wird wohl auch geglaubt, dass die durch solche Theorien des Geistes implizierten, absolut nicht-phänomenalen Explanantia für uns epistemisch zugänglich und paradoxerweise zugleich bewusstseinsunabhängig sein können. Dieser Widerspruch offenbart, dass solche Auffassungen des Mentalen an den (ihnen zugrundeliegenden) Problemen der phänomenalen Indifferenz und des phänomenalen Antirealismus' scheitern müssen.

Mit Chalmers lässt sich deshalb abschliessend konstatieren, dass wir beim hard problem einen Schritt weiter kommen, wenn wir es schaffen, die Hoffnung loszulassen, Bewusstsein auf absolut nicht-phänomenale Eigenschaften reduzieren zu können:

We can give up on the project of trying to explain the existence of consciousness wholly in terms of something more basic, and instead admit it as fundamental $[\ldots] \cdot{ }^{160}$

157 Ebd.

$15^{8}$ Ebd. [Hervorhebung E. E.].

159 Searle, John R. / [Übers.: Gavagai, Harvey]: Die Wiederentdeckung des Geistes, München: Artemis-Verlag \& Winkler [zuerst 1992, engl.]/1993, S. 26. Das heisst, das Ideal einer Ontologie, die das Explanandum des hard problem eigentlich gar nicht berücksichtigen darf.

16o Chalmers, David J.: The conscious mind. In search of a fundamental theory, New York: Oxford University Press 1996, S. 213. 